\title{
Smooth Dense Subalgebras and Fourier Multipliers on Compact Quantum Groups
}

\author{
Rauan Akylzhanov, Shahn Majid, Michael Ruzhansky iD \\ Imperial College London, London, UK. E-mail: r.akylzhanov@imperial.ac.uk
}

Received: 31 July 2017 / Accepted: 3 June 2018

Published online: 13 August 2018 - (C) The Author(s) 2018

\begin{abstract}
We define and study dense Frechet subalgebras of compact quantum groups realised as smooth domains associated with a Dirac type operator with compact resolvent. Further, we construct spectral triples on compact matrix quantum groups in terms of Clebsch-Gordon coefficients and the eigenvalues of the Dirac operator $\mathcal{D}$. Grotendieck's theory of topological tensor products immediately yields a Schwartz kernel theorem for linear operators on compact quantum groups and allows us to introduce a natural class of pseudo-differential operators on them. It is also shown that regular pseudo-differential operators are closed under compositions. As a by-product, we develop elements of the distribution theory and corresponding Fourier analysis. We give applications of our construction to obtain sufficient conditions for $L^{p}-L^{q}$ boundedness of coinvariant linear operators. We provide necessary and sufficient conditions for algebraic differential calculi on Hopf subalgebras of compact quantum groups to extend to our proposed smooth subalgebra $C_{\mathcal{D}}^{\infty}$. We check explicitly that these conditions hold true on the quantum $\mathrm{SU}_{2}^{\mathrm{q}}$ for both its 3-dimensional and 4-dimensional calculi.
\end{abstract}

\section{Introduction}

In [HL36] Hardy and Littlewood proved the following generalisation of the Plancherel's identity on the circle $\mathbb{T}$, namely

$$
\sum_{m \in \mathbb{Z}}(1+|m|)^{p-2}|\widehat{f}(m)|^{p} \leq C_{p}\|f\|_{L^{p}(\mathbb{T})}^{p}, \quad 1<p \leq 2
$$

The first and third authors were supported in parts by the EPSRC Grant EP/R003025/1 and by the Leverhulme Grant RPG-2017-151. The first author was also partially supported by the Simons-Foundation Grant 346300 and the Polish Government MNiSW 2015-2019 matching fund. No new data was created or generated during the course of this research. 
Hewitt and Ross [HR74] generalised this to the setting of compact abelian groups. Recently, the inequality has been extended [ANR15a] to compact homogeneous manifolds. In particular, on a compact Lie group $G$ of topological dimension $n$, the result can be written as

$$
\sum_{\pi \in \widehat{G}} d_{\pi}^{p\left(\frac{2}{p}-\frac{1}{2}\right)}\langle\pi\rangle^{n(p-2)}\|\widehat{f}(\pi)\|_{\mathrm{HS}}^{p} \leq C_{p}\|f\|_{L^{p}(G)}^{p},
$$

where $\langle\pi\rangle$ are obtained from eigenvalues of the Laplace operator $\Delta_{G}$ on $G$ by

$$
\sqrt{I-\Delta_{G}} \pi_{i j}=\langle\pi\rangle \pi_{i j}, \quad i, j=1, \ldots, d_{\pi} .
$$

In [You08] the Hardy-Littlewood inequality has been extended to compact matrix quantum groups of Kac type. For this purpose, the author introduced a natural length function and extended the notion of 'rapid decay' to compact matrix quantum groups of Kac type. On the other hand, the inequality (1.2) on a compact Lie group $G$ can be given a differential formulation as

$$
\left\|\mathscr{F}_{G}\left(1-\Delta_{G}\right)^{n\left(\frac{1}{2}-\frac{1}{p}\right)} f\right\|_{\ell^{p}(\widehat{G})} \leq C_{p}\|f\|_{L^{p}(G)},
$$

where $\Delta_{G}$ is the Laplacian on $G$ and $\mathscr{F}_{G}$ is the group Fourier transform. In the view of [Lic90], the operator $I-\Delta_{G}$ is the square of the spinor Dirac operator restricted to smooth functions on $G$. Thus, one can view the identity as associated to a 'Dirac-like' operator understood broadly.

The Dirac operator was first introduced in 1928 by Paul Dirac to describe the evolution of fermions and bosons and plays an essential role in mathematical physics and representation theory. The geometric Dirac operator $D$ can be constructed on an arbitrary spin Riemannian manifold $(M, g)$ and Alain Connes showed [Con13] that most of the geometry of $(M, g)$ can be reconstructed from the Dirac operator characterised abstractly as a 'spectral triple' $\left(C^{\infty}(M), D, L_{\text {spin }}^{2}(M)\right)$. The axioms of a spectral triple in Connes' sense come from KO-homology. However, it is known that $q$-deformed quantum groups and homogeneous spaces do not fit into Connes axiomatic framework [Con95] if one wants to have the correct classical limit and various authors have considered modification of the axioms. Another problem is that of 'geometric realisation' where a given spectral triple operator (understood broadly) should ideally have an interpretation as built from a spin connection and Clifford action on a spinor bundle. A unified algebrogeometric approach to this has been proposed in [BM15], starting with a differential algebra structure on a possibly noncommutative 'coordinate algebra' and building up the geometry layer by layer so as to arrive at a noncommutative-geometrically realised $D$ as an endpoint.

Compact quantum groups are quantisations of Poisson Lie groups, and it is natural to expect that every compact quantum group should possess a spectral triple $(\mathcal{A}, \mathcal{H}, \mathcal{D})$ by a quantisation process of some sort. Following an approach suggested by [CL01], Chakraborty and Pal constructed [CP08] a spectral triple on the quantum $\mathrm{SU}_{2}^{\mathrm{q}}$. A Dirac operator agreeing with a real structure on $\mathrm{SU}_{2}^{\mathrm{q}}$ has been suggested in [DLS $\left.{ }^{+} 05\right]$, which required a slight modification on the spectral triple axioms. More recently, inspired in part by [Fio98], Nesheveyev and Tuset constructed [NT10] spectral triples on the $q$-deformation $G_{q}$ of a compact simply connected Lie group $G$. To the best of our knowledge, it seems to be an open question whether there exists a spectral triple on an arbitrary compact quantum groups. And there remains the question of linking proposed spectral triples to the geometric picture. At the root of this problem is how to marry the 
analytic considerations of compact quantum groups to the differential-algebraic notion of differential calculus in the more constructive approach.

An outline of the results is as follows. After the set-up in Sect. 2 of quantum group Fourier transform, Paley-type inequalities in compact quantum groups $\mathbb{G}$ of Kac type are developed in Sect. 3 following the classical case in [ANR15a]. Section 4 studies left Fourier multipliers $A$ (i.e. translation coinvariant operators) with associated symbol $\sigma_{A}$. In Sect. 5 we introduce a 'Dirac operator' $\mathcal{D}: L^{2}(\mathbb{G}) \rightarrow L^{2}(\mathbb{G})$ defined by a sequence $\left\{\lambda_{\pi}\right\}$ of eigenvalues according to the Peter-Weyl decomposition of $\mathbb{G}$. In Theorem 5.1 we obtain a formal version of Hardy-Littlewood inequaity (1.2) by applying Paley inequality (3.2) and write this in a differential form formulation

$$
\left\|\mathscr{F}_{\mathbb{G}}|\mathcal{D}|^{\beta\left(\frac{1}{2}-\frac{1}{p}\right)} f\right\|_{\ell^{p}(\widehat{\mathbb{G}})} \leq C_{p}\|f\|_{L^{p}(\mathbb{G})}, \quad 1<p \leq 2,
$$

where an unbounded $\mathcal{D}: L^{2}(\mathbb{G}) \rightarrow L^{2}(\mathbb{G})$ is defined by $\mathcal{D} \pi=\lambda_{\pi} \pi$ and $|\mathcal{D}|^{-\beta}$ is trace class.

This goes some way towards $\left(\mathbb{G}, L^{2}(\mathbb{G}), \mathcal{D}\right)$ as a spectral triple in the sense of Alain Connes, this being the case if $\mathcal{D}$ has bounded commutators. We analyse in Theorem 5.3 when $\mathcal{D}$ defined by $\left\{\lambda_{\pi}\right\}$ is an actual spectral triple in the case of compact matrix quantum group in the sense of Woronowicz [Wor87]. Theorem 5.3 is then illustrated on the example of [CP08] on $\mathrm{SU}_{2}^{\mathrm{q}}$ with eigenvalues $\pm(2 l+1)$ in the spin $l$ part of the decomposition.

Section 6 studies smooth domains $C_{\mathcal{D}}^{\infty}=\bigcap_{\alpha>0} \operatorname{Dom}\left(|\mathcal{D}|^{\alpha}\right)$ associated with Dirac operator $\mathcal{D}$ on compact quantum groups. We study elements of distributions and rapid decay using $C_{\mathcal{D}}^{\infty}(\mathbb{G})$. Grotendieck's theory of topological tensor products immediately yields a Schwartz kernel theorem for linear operators on $C_{\mathcal{D}}^{\infty}(\mathbb{G})$ and allows us to introduce a natural class of pseudo-differential operators in this context. In Theorem 6.13 the Schwartz kernel $K_{A B}$ of composition of two regular pseudo-differential operators $A, B: C_{\mathcal{D}}^{\infty} \rightarrow C_{\mathcal{D}}^{\infty}$ is computed in terms of the kernels $K_{A}$ and $K_{B}$. In Theorem 6.14 we show that regular pseudo-differential operators acting via right-convolution kernel can be represented by their global symbols (see Definition 6.11). A similar construction of smooth domains for general operators in Hilbert spaces has been carried out in [RT16].

Section 7 looks at how this notion of $C_{\mathcal{D}}^{\infty}$ relates to the algebraic notion of differential 1-forms in the algebraic side of noncommutative differential geometry. We show that both the standard 3D left covariant and 4D bi-covariant differential calculi on $\mathbb{C}\left[\mathrm{SU}_{2}^{\mathrm{q}}\right]$ in [Wor89] extend to $C_{\mathcal{D}}^{\infty}$ if we take $\mathcal{D}$ with eigenvalues $\pm[2 l+1]_{q}$ where $[n]_{q}=\frac{q^{n}-q^{-n}}{q-q^{-1}}$ is a $q$-integer. Thus our approach to 'smooth functions' is compatible with these $q$ differential calculi, marrying the analytic and algebraic approaches. This $q$-deformed choice of $\mathcal{D}$ no longer obeys the bounded commutators condition for a spectral triple but is a natural $q$-deformation of our previous choice. On the other hand it is more closely related to the natural $q$-geometrically-realised Dirac [Maj03] and square root of a Laplace [Maj15] operators on $\mathrm{SU}_{2}^{\mathrm{q}}$ which similarly have eigenvalues modified via $q$-integers.

The authors wish to thank Yulia Kuznetsova for her advice and comments. We also want to express our gratitude to the anonymous referees for their helpful suggestions.

\section{Preliminaries}

The notion of compact quantum groups has been introduced by Woronowicz in [Wor87]. Here we adopt the defintion from [Wor98]. 
Definition 2.1. A compact quantum group is a pair $(\mathbb{G}, \Delta)$ where $\mathbb{G}$ is a unital $C^{*}$ algebra, $\Delta: \mathbb{G} \rightarrow \mathbb{G} \otimes \mathbb{G}$ is a unital, $\star$-homomorphic map which is coassociative, i.e.

$$
\left(\Delta \otimes \operatorname{Id}_{\mathbb{G}}\right) \circ \Delta=\left(\operatorname{Id}_{\mathbb{G}} \otimes \Delta\right) \circ \Delta
$$

and

$$
\overline{\operatorname{span}\left\{\left(\operatorname{Id}_{\mathbb{G}} \otimes \mathbb{G}\right) \Delta(\mathbb{G})\right\}}=\overline{\operatorname{span}\left\{\left(\mathbb{G} \otimes \operatorname{Id}_{\mathbb{G}}\right) \Delta(\mathbb{G})\right\}}=\mathbb{G} \otimes \mathbb{G},
$$

where $\mathbb{G} \otimes \mathbb{G}$ is a minimal $C^{*}$-tensor product.

The map $\Delta$ is called the coproduct of $\mathbb{G}$ and it induces the convolution on the predual $L^{1}(\mathbb{G})$,

$$
\lambda * \mu:=(\lambda \otimes \mu) \circ \Delta, \quad \lambda, \mu \in L^{1}(\mathbb{G}) .
$$

Definition 2.2. Let $(\mathbb{G}, \Delta)$ be a compact quantum group. A finite-dimensional representation $\pi$ of $(\mathbb{G}, \Delta)$ is a matrix $\left[\pi_{i j}\right]$ in $M_{n}(\mathbb{G})$ for some $n$ such that

$$
\Delta \pi_{i j}=\sum_{k=1}^{n} \pi_{i k} \otimes \pi_{k j}
$$

for all $i, j=1, \ldots, n$. We denote by $\widehat{\mathbb{G}}$ the set of all finite-dimensional irreducible unitary representations of $(\mathbb{G}, \Delta)$.

Here we denote by $M_{n}(\mathbb{G})$ the set of $n$-dimensional matrices with entries in $\mathbb{G}$. Let $\mathbb{C}[\mathbb{G}]$ denote the Hopf subalgebra space of $\mathbb{G}$ spanned by the matrix elements $\pi_{i j}$ of finite-dimensional unitary representations $\pi$ of $(\mathbb{G}, \Delta)$. It can be shown [MVD98, Proposition 7.1, Theorem 7.6] that $\mathbb{C}[\mathbb{G}]$ is a Hopf $*$-algebra dense in $\mathbb{G}$. Every element $f \in \mathbb{C}[\mathbb{G}]$ can be expanded in a finite sum

$$
f=\sum_{\pi \in I_{f}} \sum_{i, j=1}^{n_{\pi}} c_{i j} \pi_{i j},
$$

where $I_{f}$ is a finite index set. It is sufficient to define the Hopf $*$-algebra structure on $\mathbb{C}[\mathbb{G}]$ on generators $\pi_{i j}$ as follows

$$
\varepsilon\left(\pi_{i j}\right)=\delta_{i j}, \quad S\left(\pi_{i j}\right)=\pi_{j i}^{*} \text { for } \pi \in \widehat{\mathbb{G}}, i, j=1, \ldots, n_{\pi},
$$

where $\varepsilon: \mathbb{C}[\mathbb{G}] \rightarrow \mathbb{C}$ is the counit and $S: \mathbb{C}[\mathbb{G}] \rightarrow \mathbb{C}[\mathbb{G}]$ is the antipode. These operations satisfy the usual compatiblity conditions with coproduct $\Delta$ and product $m_{\mathbb{G}}$.

Every compact quantum group possesses [DK94] a functional $h$ on $\mathbb{G}$ called the Haar state such that

$$
\left(h \otimes \operatorname{Id}_{\mathbb{G}}\right) \circ \Delta(a)=h(a) 1=\left(\operatorname{Id}_{\mathbb{G}} \otimes h\right) \circ \Delta(a) .
$$

For every $\pi \in \widehat{\mathbb{G}}$ there exists a positive invertible matrix $Q^{\pi} \in \mathbb{C}^{n_{\pi} \times n_{\pi}}$ which is a unique intertwiner in $\operatorname{Hom}\left(\pi, \pi^{*}\right)$ such that

$$
\operatorname{Tr} Q^{\pi}=\operatorname{Tr}\left(Q^{\pi}\right)^{-1}>0 .
$$

We can always diagonalize matrix $Q^{\pi}$ and therefore we shall write

$$
Q^{\pi}=\operatorname{diag}\left(q_{1}^{\pi}, \ldots, q_{n_{\pi}}^{\pi}\right) .
$$


It follows from (2.2) that

$$
\sum_{i=1}^{n_{\pi}} q_{i}^{\pi}=\sum_{i=1}^{n_{\pi}} \frac{1}{q_{i}^{\pi}}=: d_{\pi}
$$

which defines the quantum dimension $d_{\pi}$ of $\pi$. If $\mathbb{G}$ is a compact quantum group of Kac type, then $d_{\pi}=n_{\pi}$. The Peter-Weyl orthogonality relations are as follows

$$
\begin{aligned}
& h\left(\left(\pi_{i j}\right)^{*} \pi_{k l}^{\prime}\right)=\delta_{\pi \pi^{\prime}} \delta_{i k} \delta_{j l} \frac{1}{d_{\pi} q_{k}^{\pi}}, \\
& h\left(\pi_{k l}\left(\pi_{i j}^{\prime}\right)^{*}\right)=\delta_{\pi \pi^{\prime}} \delta_{i k} \delta_{j l} \frac{q_{j}^{\pi}}{d_{\pi}} .
\end{aligned}
$$

The quantum Fourier transform $\mathscr{F}_{\mathbb{G}}: L^{1}(\mathbb{G}) \rightarrow L^{\infty}(\widehat{\mathbb{G}})$ is given by

$$
\widehat{f}(\pi)_{i j}=h\left(f \pi_{j i}^{*}\right), \quad i, j=1, \ldots, n_{\pi},
$$

where $L^{1}(\mathbb{G})$ and $L^{\infty}(\widehat{\mathbb{G}})$ are defined below. The inverse Fourier transform $\mathscr{F}_{\mathbb{G}}^{-1}$ is given by

$$
f=\sum_{\pi \in \widehat{\mathbb{G}}} d_{\pi} \operatorname{Tr}\left(\left(Q^{\pi}\right)^{-1} \pi \widehat{f}(\pi)\right)=\sum_{\pi} \sum_{i, j=1}^{n_{\pi}} \frac{d_{\pi}}{q_{i}^{\pi}} \pi_{j i} \hat{f}(\pi)_{i j} .
$$

From this it follows that $\left\{\sqrt{d_{\pi} q_{i}^{\pi}} \pi_{i j}: \pi \in \widehat{\mathbb{G}}, 1 \leq i, j \leq n_{\pi}\right\}$ is an orthonormal basis in $L^{2}(\mathbb{G})$. The Plancherel identity takes the form

$$
(f, g)_{L^{2}(\mathbb{G})}=\sum_{\pi \in \widehat{\mathbb{G}}} d_{\pi} \operatorname{Tr}\left(\left(Q^{\pi}\right)^{-1} \widehat{f}(\pi) \widehat{g}(\pi)^{*}\right) .
$$

We denote by $\mathcal{C}(\pi)$ the coefficients subcoalgebra

$$
\mathcal{C}(\pi)=\operatorname{span}\left\{\pi_{i j}\right\}_{i, j=1}^{n_{\pi}} .
$$

The Peter-Weyl decomposition on the Hopf algebra $\mathbb{C}[\mathbb{G}]$ is of the form

$$
\mathbb{C}[\mathbb{G}]=\bigoplus_{\pi \in \widehat{\mathbb{G}}} \mathcal{C}(\pi) \text {. }
$$

Let $L^{2}(\mathbb{G})$ be the GNS-Hilbert space associated with the Haar weight $h$. We denote by $L^{\infty}(\mathbb{G})$ the universal von Neumann enveloping algebra of $\mathbb{G}$. The coproduct $\Delta$ and the Haar weight $h$ can be uniquely extended to $L^{\infty}(\mathbb{G})$. In general, there are two approaches to locally compact quantum groups: $C^{*}$-algebraic and von Neumann-algebraic.

Let $\psi$ be a normal semi-finite weight on the commutant $\left[L^{\infty}(\mathbb{G})\right]^{\prime}$ of the von Neumann algebra $L^{\infty}(\mathbb{G})$. Let $L^{1}(\mathbb{G}, \psi)$ be the set of all closed, densely defined operators $A$ with polar decomposition $A=u|A|$ such that there exists positive $\phi \in L^{\infty}(\mathbb{G})_{*}$ and its spatial derivative $\frac{d \phi}{d \psi}=|A|$. Setting $\|A\|_{L^{1}(\mathbb{G})}=\phi(1)=\|\phi\|_{L^{\infty}(\mathbb{G})_{*}}$ yields an isometric isomorphism between $L^{1}(\mathbb{G}, \psi)$ and $L^{\infty}(\mathbb{G})_{*}$. Analogously, we denote by $L^{p}(\mathbb{G}, \psi)$ the set of all closed, densely defined operators $x$ such that there exists $\phi \in L^{1}(\mathbb{G}, \psi)$ such that $|A|^{p}=\frac{d \phi}{d \psi}$ with the $L^{p}$-norm given by $\|A\|_{L^{p}(\mathbb{G})}=\phi(1)^{\frac{1}{p}}$. These spaces are isometrically isomorphic to the Haagerup $L^{p}$-spaces [Haa79] and are thus independent of the choice of $\psi$.

One can introduce the Lebesgue space $\ell^{p}(\widehat{\mathbb{G}})$ on the dual $\widehat{\mathbb{G}}$ as follows 
Definition 2.3. We shall denote by $\ell^{p}(\widehat{\mathbb{G}})$ the space of sequences $\{\sigma(\pi)\}_{\pi \in \widehat{\mathbb{G}}}$ endowed with the norm

$$
\|\sigma\|_{\ell p(\widehat{\mathbb{G}})}=\left(\sum_{\pi \in \widehat{\mathbb{G}}} d_{\pi} n_{\pi}\left(\frac{\|\sigma(\pi)\|_{\mathrm{HS}}}{\sqrt{n_{\pi}}}\right)^{p}\right)^{\frac{1}{p}}, \quad 1 \leq p<\infty .
$$

Here by the Hilbert-Schmidt norm we mean

$$
\|\sigma(\pi)\|_{\mathrm{HS}}^{2}=\operatorname{Tr}\left(Q^{\pi}\right)^{-1} \sigma(\pi) \sigma(\pi)^{*} .
$$

For $p=\infty$, we write $L^{\infty}(\widehat{\mathbb{G}})$ for the space of all $\sigma$ such that

$$
\|\sigma\|_{L^{\infty}(\widehat{\mathbb{G}})}:=\sup _{\pi \in \widehat{\mathbb{G}}} \frac{\|\sigma(\pi)\|_{\mathrm{HS}}}{\sqrt{n_{\pi}}}<\infty .
$$

It can be shown that these are interpolation spaces in analogy to a similar family of spaces on the unitary dual of compact topological groups. The latter spaces were introduced in [RT10]. In this notation we can rewrite (2.5) as

$$
\|f\|_{L^{2}(\mathbb{G})}^{2}=\sum_{\pi \in \widehat{\mathbb{G}}} d_{\pi}\|\widehat{f}\|_{\mathrm{HS}}^{2} .
$$

It can be shown that $\mathscr{F}_{\mathbb{G}}: f \mapsto \widehat{f}=\{\widehat{f}(\pi)\}$ is a contraction, i.e.

$$
\|\widehat{f}(\pi)\|_{\mathrm{op}} \leq\|f\|_{L^{1}(\mathbb{G})}, \quad \pi \in \widehat{\mathbb{G}} .
$$

Using the Hilbert-Schmidt norm and unitarity $\pi \pi^{*}=$ Id of $\pi$ 's, one can show

$$
\|\widehat{f}(\pi)\|_{\mathrm{HS}} \leq \sqrt{n_{\pi}}\|f\|_{L^{1}(\mathbb{G})}, \quad \pi \in \widehat{\mathbb{G}} .
$$

Hence, by the interpolation theorem for $1<p \leq 2$ we obtain two versions of HausdorffYoung inequality

$$
\begin{aligned}
& \left(\sum_{\pi \in \widehat{\mathbb{G}}} d_{\pi}\|\widehat{f}(\pi)\|_{\ell p\left(\mathbb{C}^{\left.n_{\pi} \times n_{\pi}\right)}\right.}^{p^{\prime}}\right)^{\frac{1}{p^{\prime}}}=:\|\widehat{f}\|_{\ell_{s c h}^{p^{\prime}(\widehat{\mathbb{G}})}} \leq\|f\|_{L^{p}(\mathbb{G})}, \\
& \left(\sum_{\pi \in \widehat{\mathbb{G}}} d_{\pi} n_{\pi}\left(\frac{\|\widehat{f}(\pi)\|_{\mathrm{HS}}}{\sqrt{n_{\pi}}}\right)^{p^{\prime}}\right)^{\frac{1}{p^{\prime}}}=:\|\widehat{f}\|_{\ell^{p^{\prime}(\widehat{\mathbb{G}})}} \leq\|f\|_{L^{p}(\mathbb{G})} .
\end{aligned}
$$

A Hausdorff-Young inequality on locally compact quantum groups has been obtained in [Coo10] and its sharpness explored in [LWW17].

We also present a version of a Marcinkiewicz interpolation theorem for linear mappings between compact quantum group $\mathbb{G}$ of Kac type and the space of matrix-valued sequences $\Sigma$ that will be realised via

$$
\Sigma:=\left\{\sigma=\{\sigma(\pi)\}_{\pi \in \widehat{\mathbb{G}}}, \sigma(\pi) \in \mathbb{C}^{n_{\pi} \times n_{\pi}}\right\} .
$$

Thus, a linear mapping $A: L^{\infty}(\mathbb{G}) \rightarrow \Sigma$ takes a function to a matrix valued sequence, i.e.

$$
f \mapsto A f=: \sigma=\{\sigma(\pi)\}_{\pi \in \widehat{G}},
$$


where

$$
\sigma(\pi) \in \mathbb{C}^{n_{\pi} \times n_{\pi}}, \pi \in \widehat{\mathbb{G}}
$$

We say that a linear operator $A$ is of strong type $(p, q)$, if for every $f \in L^{p}(\mathbb{G})$, we have Af $\in \ell^{q}(\widehat{\mathbb{G}}, \Sigma)$ and

$$
\|A f\|_{\ell q(\widehat{\mathbb{G}}, \Sigma)} \leq M\|f\|_{L^{p}(G)},
$$

where $M$ is independent of $f$, and the space $\ell^{q}(\widehat{\mathbb{G}}, \Sigma)$ defined by the norm

$$
\|\sigma\|_{\ell^{q}(\widehat{\mathbb{G}}, \Sigma)}:=\left(\sum_{\pi \in \widehat{\mathbb{G}}} n_{\pi}^{p\left(\frac{2}{p}-\frac{1}{2}\right)}\|\sigma(\pi)\|_{\mathrm{HS}}^{p}\right)^{\frac{1}{p}}
$$

as in (2.7). The least $M$ for which this is satisfied is taken to be the strong $(p, q)$-norm of the operator $A$. i.e.

Denote the distribution functions of $f$ and $\sigma$ by $\mu_{\mathbb{G}}(t ; f)$ and $v_{\widehat{\mathbb{G}}}(y ; \sigma)$, respectively,

$$
\begin{aligned}
& \mu_{\mathbb{G}}(t ; f):=h\left(E_{(t,+\infty)}(|f|)\right), \quad t>0, \\
& \nu_{\widehat{\mathbb{G}}}(y ; \sigma):=\sum_{\substack{\pi \in \widehat{\mathbb{G}} \\
\frac{\|\sigma(\pi)\| \mathrm{HS}}{\sqrt{n_{\pi}}} \geq y}} n_{\pi}^{2}, \quad y>0 .
\end{aligned}
$$

Then

$$
\begin{aligned}
\|f\|_{L^{p}(\mathbb{G})}^{p} & =p \int_{0}^{+\infty} t^{p-1} \mu_{\mathbb{G}}(t ; f) d t, \\
\|\sigma\|_{\ell^{q}(\widehat{\mathbb{G}}, \Sigma)}^{q} & =\sum_{\pi \in \widehat{\mathbb{G}}} n_{\pi}^{2}\left(\frac{\|\sigma(\pi)\|_{\mathrm{H},}}{\sqrt{n_{\pi}}}\right)^{q}=q \int_{0}^{+\infty} y^{q-1} \nu_{\widehat{\mathbb{G}}}(y ; \sigma) d y .
\end{aligned}
$$

A linear operator $A: L^{\infty}(\mathbb{G}) \rightarrow \Sigma$ satisfying

$$
\nu_{\widehat{G}}(y ; A f) \leq\left(\frac{M}{y}\|f\|_{L^{p}(G)}\right)^{q}
$$

is said to be of weak type $(p, q)$; the least value of $M$ in $(2.18)$ is called weak $(p, q)$ norm of $A$.

Every operation of strong type $(p, q)$ is also of weak type $(p, q)$, since

$$
y\left(v_{\widehat{\mathbb{G}}}(y ; A f)\right)^{\frac{1}{q}} \leq\|A f\|_{L^{q}(\widehat{\mathbb{G}})} \leq M\|f\|_{L^{p}(\mathbb{G})} .
$$

The classical Marcinkiewicz interpolation theorem [Fol99, Theorem 6.28] has a natural analogue [ANR16, Theorem 6.1] for compact Lie groups. As a special case of [BC12, Theorem 2.1] we immediately obtain 
Theorem 2.4. Let $1 \leq p_{1}<p<p_{2}<\infty$. Suppose that a linear operator $A$ from $\mathrm{七}^{\infty}(\mathbb{G})$ to $\Sigma$ is simultaneously of weak types $\left(p_{1}, p_{1}\right)$ and $\left(p_{2}, p_{2}\right)$, with norms $M_{1}$ and $M_{2}$, respectively, i.e.

$$
\begin{aligned}
& v_{\widehat{\mathbb{G}}}(y ; A f) \leq\left(\frac{M_{1}}{y}\|f\|_{L^{p_{1}}(G)}\right)^{p_{1}}, \\
& v_{\widehat{\mathbb{G}}}(y ; A f) \leq\left(\frac{M_{2}}{y}\|f\|_{L^{p_{2}}(G)}\right)^{p_{2}} .
\end{aligned}
$$

Then for any $p \in\left(p_{1}, p_{2}\right)$ the operator $A$ is of strong type $(p, p)$ and we have

$$
\|A f\|_{\ell^{p}(\widehat{\mathbb{G}}, \Sigma)} \leq M_{1}^{1-\theta} M_{2}^{\theta}\|f\|_{L^{p}(G)}, \quad 0<\theta<1,
$$

where

$$
\frac{1}{p}=\frac{1-\theta}{p_{1}}+\frac{\theta}{p_{2}} .
$$

A different special case of [BC12, Theorem 2.1] has been presented in [You08].

\section{Hausdorff-Young-Paley Inequalities}

A Paley-type inequality for the group Fourier transform on commutative compact quantum group $\mathbb{G}=C(G)$ has been obtained in [ANR15a]. Here we give an analogue of this inequality on an arbitrary compact quantum group $\mathbb{G}$ of Kac type.

Theorem 3.1 (Paley-type inequality). Let $1<p \leq 2$ and let $\mathbb{G}$ be a compact quantum group of Kac type. If $\varphi(\pi)$ is a positive sequence over $\widehat{\mathbb{G}}$ such that

$$
M_{\varphi}:=\sup _{y>0} y \sum_{\substack{\pi \in \widehat{\mathbb{G}} \\ \varphi(\pi) \geq y}} n_{\pi}^{2}<\infty
$$

is finite, then we have

$$
\left(\sum_{\pi \in \widehat{\mathbb{G}}} n_{\pi}^{2}\left(\frac{\|\widehat{f}(\pi)\|_{\mathrm{HS}}}{\sqrt{n_{\pi}}}\right)^{p} \varphi(\pi)^{2-p}\right)^{\frac{1}{p}} \lesssim M_{\varphi}^{\frac{2-p}{p}}\|f\|_{L^{p}(\mathbb{G})} .
$$

The main tool in the proof of Theorem 3.1 is a noncommutative version of the Marcinkiewicz interpolation Theorem 2.4). Following the classical case in [ANR16] and [ANR15a], Youn obtained [You08] a different version of Paley type inequalities on compact quantum groups of Kac type.

Theorem 3.2 (Hausdorff-Young-Paley inequality). Let $1<p \leq b \leq p^{\prime}<\infty$ and let $\mathbb{G}$ be a compact quantum group of Kac type. If a positive sequence $\varphi(\pi), \pi \in \widehat{\mathbb{G}}$, satisfies the condition

$$
M_{\varphi}:=\sup _{y>0} y \sum_{\substack{\pi \in \widehat{\mathbb{G}} \\ \varphi(\pi) \geq y}} n_{\pi}^{2}<\infty,
$$

then we have

$$
\left(\sum_{\pi \in \widehat{\mathbb{G}}} n_{\pi}^{2}\left(\frac{\|\widehat{f}(\pi)\|_{\mathrm{HS}}}{\sqrt{n_{\pi}}} \varphi(\pi)^{\frac{1}{b}-\frac{1}{p^{\prime}}}\right)^{b}\right)^{\frac{1}{b}} \lesssim M_{\varphi}^{\frac{1}{b}-\frac{1}{p^{\prime}}}\|f\|_{L^{p}(\mathbb{G})} .
$$


Further, we recall a result on the interpolation of weighted spaces from [BL76]:

Theorem 3.3 (Interpolation of weighted spaces). Let us write $d \mu_{0}(x)=\omega_{0}(x) d \mu(x)$, $d \mu_{1}(x)=\omega_{1}(x) d \mu(x)$, and write $L^{p}(\omega)=L^{p}(\omega d \mu)$ for the weight $\omega$.

Suppose that $0<p_{0}, p_{1}<\infty$. Then

$$
\left(L^{p_{0}}\left(\omega_{0}\right), L^{p_{1}}\left(\omega_{1}\right)\right)_{\theta, p}=L^{p}(\omega)
$$

where $0<\theta<1, \frac{1}{p}=\frac{1-\theta}{p_{0}}+\frac{\theta}{p_{1}}$, and $\omega=w_{0}^{p \frac{1-\theta}{p_{0}}} w_{1}^{p \frac{\theta}{p_{1}}}$.

From this, interpolating between the Paley-type inequality (3.2) in Theorem 3.1 and Hausdorff-Young inequality (2.13), we obtain Theorem 3.2. Hence, we concentrate on proving Theorem 3.1. The proof of Theorem 3.1 is an adaptation of the techniques used in [ANR15a].

Proof of Theorem 3.1. Let $\mu$ give measure $\varphi^{2}(\pi) n_{\pi}^{2}, \pi \in \widehat{\mathbb{G}}$ to the set consisting of the single point $\{\pi\}, \pi \in \mathbb{G}$, and measure zero to a set which does not contain any of these points, i.e.

$$
\mu\{\pi\}:=\varphi^{2}(\pi) n_{\pi}^{2} .
$$

We define the space $L^{p}(\mathbb{G}, \mu), 1 \leq p<\infty$, as the space of complex (or real) sequences $a=\left\{a_{l}\right\}_{l \in \widehat{\mathbb{G}}}$ such that

$$
\|a\|_{L^{p}(\mathbb{G}, \mu)}:=\left(\sum_{l \in \widehat{\mathbb{G}}}\left|a_{l}\right|^{p} \varphi^{2}(\pi) n_{\pi}^{2}\right)^{\frac{1}{p}}<\infty .
$$

We will show that the sub-linear operator

$$
T: L^{p}(\mathbb{G}) \ni f \mapsto T f=a=\left\{\frac{\|\widehat{f}(\pi)\|_{\mathrm{HS}}}{\sqrt{n_{\pi}} \varphi(\pi)}\right\}_{\pi \in \widehat{\mathbb{G}}} \in L^{p}(\widehat{\mathbb{G}}, \mu)
$$

is well-defined and bounded from $L^{p}(\mathbb{G})$ to $L^{p}(\widehat{\mathbb{G}}, \mu)$ for $1<p \leq 2$. In other words, we claim that we have the estimate

$$
\|T f\|_{L^{p}(\widehat{\mathbb{G}}, \mu)}=\left(\sum_{\pi \in \mathbb{G}}\left(\frac{\|\widehat{f}(\pi)\|_{\mathrm{HS}}}{\sqrt{n_{\pi}} \varphi(\pi)}\right)^{p} \varphi^{2}(\pi) n_{\pi}^{2}\right)^{\frac{1}{p}} \lesssim K_{\varphi}^{\frac{2-p}{p}}\|f\|_{L^{p}(\mathbb{G})},
$$

which would give (3.2) and where we set $K_{\varphi}:=\sup _{y>0} y \sum_{\substack{\pi \in \mathbb{G} \\ \varphi(\pi) \geq y}} n_{\pi}^{2}$. We will show that $A$ is of weak type $(2,2)$ and of weak-type $(1,1)$. More precisely, with the distribution function

$$
\nu_{\mathbb{G}}(y ; T f)=\sum_{\substack{\pi \in \widehat{\mathbb{G}} \\|T f(\pi)| \geq y}} \varphi^{2}(\pi) n_{\pi}^{2}
$$

we show that

$$
\nu_{\mathbb{G}}(y ; T f) \leq\left(\frac{M_{2}\|f\|_{L^{2}(\mathbb{G})}}{y}\right)^{2} \quad \text { with norm } M_{2}=1,
$$




$$
v_{\mathbb{G}}(y ; T f) \leq \frac{M_{1}\|f\|_{L^{1}(\mathbb{G})}}{y} \quad \text { with norm } M_{1}=K_{\varphi} .
$$

Then (3.2) will follow by the Marcinkiewicz interpolation Theorem 2.4). Now, to show (3.6), using Plancherel's identity (2.10), we get

$$
\begin{aligned}
y^{2} v_{\mathbb{G}}(y ; T f) \leq\|T f\|_{L^{2}(\mathbb{G}, \mu)}^{2} & =\sum_{\pi \in \widehat{\mathbb{G}}}\left(\frac{\|\widehat{f}(\pi)\|_{\mathrm{HS}}}{\sqrt{n_{\pi}} \varphi(\pi)}\right)^{2} \varphi^{2}(\pi) n_{\pi}^{2} \\
& =\sum_{\pi \in \widehat{\mathbb{G}}} n_{\pi}\|\widehat{f}(\pi)\|_{\mathrm{HS}}^{2}=\|\widehat{f}\|_{\ell^{2}(\widehat{\mathbb{G}})}^{2}=\|f\|_{L^{2}(\mathbb{G})}^{2} .
\end{aligned}
$$

Thus, $T$ is of type $(2,2)$ with norm $M_{2} \leq 1$. Further, we show that $T$ is of weak-type $(1,1)$ with norm $M_{1}=C$; more precisely, we show that

$$
\nu_{\mathbb{G}}\left\{\pi \in \widehat{\mathbb{G}}: \frac{\|\widehat{f}(\pi)\|_{\mathrm{HS}}}{\sqrt{n_{\pi}} \varphi(\pi)}>y\right\} \lesssim K_{\varphi} \frac{\|f\|_{L^{1}(\mathbb{G})}}{y} .
$$

The left-hand side here is the weighted sum $\sum \varphi^{2}(\pi) n_{\pi}^{2}$ taken over those $\pi \in \widehat{\mathbb{G}}$ for which $\frac{\|\widehat{f}(\pi)\|_{\mathrm{HS}}}{\sqrt{n_{\pi}} \varphi(\pi)}>y$.

From definition of the Fourier transform it follows that

$$
\|\widehat{f}(\pi)\|_{\mathrm{HS}} \leq \sqrt{n_{\pi}}\|f\|_{L^{1}(\mathbb{G})} .
$$

Therefore, we have

$$
y<\frac{\|\widehat{f}(\pi)\|_{\mathrm{HS}}}{\sqrt{n_{\pi}} \varphi(\pi)} \leq \frac{\|f\|_{L^{1}(\mathbb{G})}}{\varphi(\pi)} .
$$

Using this, we get

$$
\left\{\pi \in \widehat{\mathbb{G}}: \frac{\|\widehat{f}(\pi)\|_{\mathrm{HS}}}{\sqrt{n_{\pi}} \varphi(\pi)}>y\right\} \subset\left\{\pi \in \widehat{\mathbb{G}}: \frac{\|f\|_{L^{1}(\mathbb{G})}}{\varphi(\pi)}>y\right\}
$$

for any $y>0$. Consequently,

$$
\mu\left\{\pi \in \widehat{\mathbb{G}}: \frac{\|\widehat{f}(\pi)\|_{\mathrm{HS}}}{\sqrt{n_{\pi}} \varphi(\pi)}>y\right\} \leq \mu\left\{\pi \in \widehat{\mathbb{G}}: \frac{\|f\|_{L^{1}(\mathbb{G})}}{\varphi(\pi)}>y\right\} .
$$

Setting $v:=\frac{\|f\|_{L^{1}(\mathbb{G})}}{y}$, we get

$$
\mu\left\{\pi \in \widehat{\mathbb{G}}: \frac{\|\widehat{f}(\pi)\|_{\mathrm{HS}}}{\sqrt{n_{\pi}} \varphi(\pi)}>y\right\} \leq \sum_{\substack{\pi \in \widehat{\mathbb{G}} \\ \varphi(\pi) \leq v}} \varphi^{2}(\pi) n_{\pi}^{2} .
$$

We claim that

$$
\sum_{\substack{\pi \in \widehat{\mathbb{G}} \\ \varphi(\pi) \leq v}} \varphi^{2}(\pi) n_{\pi}^{2} \lesssim K_{\varphi} v
$$


In fact, we have

$$
\sum_{\substack{\pi \in \widehat{\mathbb{G}} \\ \varphi(\pi) \leq v}} \varphi^{2}(\pi) n_{\pi}^{2}=\sum_{\substack{\pi \in \widehat{\mathbb{G}} \\ \varphi(\pi) \leq v}} n_{\pi}^{2} \int_{0}^{\varphi^{2}(\pi)} d \tau
$$

We can interchange sum and integration to get

$$
\sum_{\substack{\pi \in \widehat{\mathbb{G}} \\ \varphi(\pi) \leq v}} n_{\pi}^{2} \int_{0}^{\varphi^{2}(\pi)} d \tau=\int_{0}^{v^{2}} d \tau \sum_{\substack{\pi \in \widehat{\mathbb{G}} \\ \tau^{\frac{1}{2}} \leq \varphi(\pi) \leq v}} n_{\pi}^{2}
$$

Further, we make a substitution $\tau=y^{2}$, yielding

$$
\int_{0}^{v^{2}} d \tau \sum_{\substack{\pi \in \widehat{\mathbb{G}} \\ \tau^{\frac{1}{2}} \leq \varphi(\pi) \leq v}} n_{\pi}^{2}=2 \int_{0}^{v} y d y \sum_{\substack{\pi \in \widehat{\mathbb{G}} \\ y \leq \varphi(\pi) \leq v}} n_{\pi}^{2} \leq 2 \int_{0}^{v} y d y \sum_{\substack{\pi \in \widehat{\mathbb{G}} \\ y \leq \varphi(\pi)}} n_{\pi}^{2} .
$$

Since

$$
y \sum_{\substack{\pi \in \widehat{\mathbb{G}} \\ y \leq \varphi(\pi)}} n_{\pi}^{2} \leq \sup _{y>0} y \sum_{\substack{\pi \in \widehat{\mathbb{G}} \\ y \leq \varphi(\pi)}} n_{\pi}^{2}=: K_{\varphi}
$$

is finite by the definition of $K_{\varphi}$, we have

$$
2 \int_{0}^{v} y d y \sum_{\substack{\pi \in \widehat{\mathbb{G}} \\ y \leq \varphi(\pi)}} n_{\pi}^{2} \lesssim K_{\varphi} v
$$

This proves (3.10). We have just proved inequalities (3.6), (3.7). Then by using Marcinkiewicz' interpolation theorem (see Theorem 2.4) with $p=1, q=2$ and $\frac{1}{p}=$ $1-\theta+\frac{\theta}{2}$ we now obtain

$$
\left(\sum_{\pi \in \widehat{\mathbb{G}}}\left(\frac{\|\widehat{f}(\pi)\|_{\mathrm{HS}}}{\sqrt{n_{\pi}} \varphi(\pi)}\right)^{p} \varphi^{2}(\pi) n_{\pi}^{2}\right)^{\frac{1}{p}}=\|T f\|_{L^{p}(\widehat{\mathbb{G}})} \lesssim K_{\varphi^{\frac{2-p}{p}}}\|f\|_{L^{p}(\mathbb{G})} .
$$

This completes the proof. 


\section{Fourier Multipliers on Compact Quantum Groups}

Definition 4.1. Let $(\mathbb{G}, \Delta)$ be a compact quantum group. A linear operator $A: \mathbb{G} \rightarrow \mathbb{G}$ is called a left Fourier multiplier if

$$
\Delta \circ A=(\operatorname{Id} \otimes A) \circ \Delta .
$$

For a compact topological group this means an operator that commutes with left translation on the group and defines a global symbol $\sigma_{A}$ of $A$.

Theorem 4.2. Let $\mathbb{G}$ be a compact quantum group and let $A: \mathbb{G} \rightarrow \mathbb{G}$ be a left Fourier multiplier. Then

$$
\widehat{A f}(\pi)=\sigma_{A}(\pi) \widehat{f}(\pi), \quad f \in L^{2}(\mathbb{G}),
$$

where $\sigma_{A}(\pi) \in \mathbb{C}^{n_{\pi} \times n_{\pi}}$ are defined by $A \pi=\pi \sigma_{A}(\pi)$.

Proof of Theorem 4.2. By the Peter-Weyl decomposition (2.6), it is sufficient to establish (4.2) on the coefficient sub-colagebra $\mathcal{C}(\pi)$. Suppose $A$ is left invariant and write

$$
A \pi_{i j}=\sum_{\pi^{\prime} \in \widehat{\mathbb{G}}} \sum_{i, k=1}^{n_{\pi}} \pi_{k l}^{\prime} c_{i j k l}^{\pi^{\prime}}
$$

for some coefficients $c_{i j k l}^{\pi^{\prime}}$. Then by left invariance,

$$
\begin{aligned}
\Delta A \pi_{i j} & =\sum_{\pi^{\prime}, k, l, m} c_{i j k l}^{\pi^{\prime}} \pi_{k m}^{\prime} \otimes \pi_{m l}^{\prime}=(\mathrm{id} \otimes A) \Delta \pi_{i j} \\
& =\sum_{m} \pi_{i m} \otimes A \pi_{m j}=\sum_{m} \sum_{l, k} C_{m j k l}^{\pi^{\prime}} \pi_{i m} \otimes \pi_{k l}^{\prime}
\end{aligned}
$$

Comparing these, by the Peter-Weyl decomposition, we see that only $\pi^{\prime}=\pi$ can contribute. Moreover, since the $\left\{\pi_{i j}\right\}$ are a basis of $C(\pi)$ we must have $c_{i j k l}^{\pi}=0$ unless $k=i$. So we have $\sum_{l, m} c_{i j i l}^{\pi} \pi_{i m} \otimes \pi_{m l}=\sum_{m} \pi_{i m} \otimes A \pi_{m j}$. Comparing these we see that

$$
A \pi_{m j}=\sum_{l} \pi_{m l} \sigma_{A}(\pi)_{l j}
$$

from some matrix $\sigma_{A}(\pi)_{l j}=c_{i j i l}^{\pi}$ which can not depend on $i$. Finally, setting $f=\pi_{k l}$ we have

$$
\widehat{\pi_{k l}}\left(\pi^{\prime}\right)_{i j}=h\left(\pi_{k l} \pi_{j i}^{\prime *}\right)=\delta_{\pi, \pi^{\prime}} \frac{q_{k}^{\pi}}{d_{\pi}} \delta_{k j} \delta_{l i}
$$

and we check that

$$
\begin{aligned}
\widehat{A \pi_{k l}}\left(\pi^{\prime}\right)_{i j} & =\sum_{m} \widehat{\pi_{k m}}\left(\pi^{\prime}\right)_{i j} \sigma_{A}(\pi)_{m l}=\delta_{\pi, \pi^{\prime}} \frac{q_{k}^{\pi}}{d_{\pi}} \delta_{k j} \sigma_{A}(\pi)_{i l}, \\
\left(\sigma_{A}\left(\pi^{\prime}\right) \widehat{\pi_{k l}}\left(\pi^{\prime}\right)\right)_{i j} & =\sum_{m} \sigma_{A}\left(\pi^{\prime}\right)_{i m} \widehat{\pi_{k l}}\left(\pi^{\prime}\right)_{m j}=\sigma_{A}(\pi)_{i l} \delta_{\pi, \pi^{\prime}} \frac{q_{k}^{\pi}}{d_{\pi}} \delta_{k j},
\end{aligned}
$$

which is the same. This proves Theorem 4.3. 
Essentially similar arguments can be found in an earlier paper by [CFK14]. Also note from the proof that the same result applies to any coinvariant linear map $A: \mathbb{C}[\mathbb{G}] \rightarrow$ $\mathbb{C}[\mathbb{G}]$. We refer to the operators $A$ acting in this way on the Fourier side as quantum Fourier multipliers. In greater generality, such result was also shown in [JNR09] for every locally compact quantum group; the authors thank the referee for pointing out this reference. In the classical situation on $\mathbb{G}=\mathbb{T}^{n}$, left Fourier multipliers are essentially operators acting via convolution with measures whose Fourier coefficients are bounded.

Let $A: \mathbb{G} \rightarrow \mathbb{G}$ be a left Fourier multiplier. We are concerned with the question of what assumptions on the symbol $\sigma_{A}$ guarantee that $A$ is bounded from $L^{p}(\mathbb{G})$ to $L^{q}(\mathbb{G})$.

Theorem 4.3. Let $\mathbb{G}$ be a compact quantum group of Kac type. Let $1<p \leq 2 \leq q<\infty$ and let $A: L^{2}(\mathbb{G}) \rightarrow L^{2}(\mathbb{G})$ be a left Fourier multiplier. Then we have

$$
\|A\|_{L^{p}(\mathbb{G}) \rightarrow L^{q}(\mathbb{G})} \lesssim \sup _{s>0}\left(\sum_{\substack{\pi \in \widehat{\mathbb{G}}_{\mathrm{op}}>s \\\left\|\sigma_{A}(\pi)\right\|_{\mathrm{op}}}} n_{\pi}^{\frac{1}{p}-\frac{1}{q}} .\right.
$$

Proof of Theorem 4.3. By definition

$$
\widehat{A f}(\pi)=\sigma_{A}(\pi) \widehat{f}(\pi) .
$$

Let us first assume that $p \leq q^{\prime}$ (where $\frac{1}{q}+\frac{1}{q^{\prime}}=1$ ). Since $q^{\prime} \leq 2$, for $f \in \mathbb{C}[\mathbb{G}]$ the Hausdorff-Young inequality gives

$$
\begin{aligned}
\|A f\|_{L^{q}(\mathbb{G})} & \leq\|\widehat{A f}\|_{\ell q^{\prime}(\widehat{\mathbb{G}})}=\left\|\sigma_{A} \widehat{f}\right\|_{\ell q^{\prime}(\widehat{\mathbb{G}})}=\left(\sum_{\pi \in \widehat{\mathbb{G}}} n_{\pi}^{2}\left(\frac{\left\|\sigma_{A}(\pi) \widehat{f}(\pi)\right\|_{\mathrm{HS}}}{\sqrt{n_{\pi}}}\right)^{q^{\prime}}\right)^{\frac{1}{q^{\prime}}} \\
& \leq\left(\sum_{\pi \in \mathbb{G}} n_{\pi}^{2}\left\|\sigma_{A}(\pi)\right\|_{\mathrm{OP}}^{q^{\prime}}\left(\frac{\|\widehat{f}(\pi)\|_{\mathrm{HS}}}{\sqrt{n_{\pi}}}\right)^{q^{\prime}}\right)^{\frac{1}{q^{\prime}}} .
\end{aligned}
$$

The case $q^{\prime} \leq\left(p^{\prime}\right)^{\prime}$ can be reduced to the case $p \leq q^{\prime}$ as follows. The $L^{p}$-duality yields

$$
\|A\|_{L^{p}(\mathbb{G}) \rightarrow L^{q}(\mathbb{G})}=\left\|A^{*}\right\|_{L^{q^{\prime}}(\mathbb{G}) \rightarrow L^{p^{\prime}}(\mathbb{G})} .
$$

The symbol $\sigma_{A^{*}}(\pi)$ of the adjoint operator $A^{*}$ equals to $\sigma_{A}^{*}(\pi)$,

$$
\sigma_{A^{*}}(\pi)=\sigma_{A}^{*}(\pi), \quad \pi \in \widehat{\mathbb{G}},
$$

and its operator norm $\left\|\sigma_{A^{*}}(\pi)\right\|_{\text {op }}$ equals to $\left\|\sigma_{A}(\pi)\right\|_{\mathrm{op}}$. Now, we are in a position to apply Theorem 3.2. Set $\frac{1}{r}=\frac{1}{p}-\frac{1}{q}$. We observe that with $\sigma(\pi):=\left\|\sigma_{A}(\pi)\right\|_{\mathrm{op}}^{r} I_{d_{\pi}}, \pi \in$ $\widehat{\mathbb{G}}$, and $b=q^{\prime}$, the assumptions of Theorem 3.2 are satisfied and we obtain

$$
\left(\sum_{\pi \in \widehat{\mathbb{G}}} n_{\pi}^{2}\left\|\sigma_{A}(\pi)\right\|_{\mathrm{Op}}^{q^{\prime}}\left(\frac{\|\widehat{f}(\pi)\|_{\mathrm{HS}}}{\sqrt{n_{\pi}}}\right)^{q^{\prime}}\right)^{\frac{1}{q^{\prime}}} \lesssim\left(\sup _{s>0} \sum_{\substack{\pi \in \widehat{\mathbb{G}}^{r}>s \\\|\sigma(\pi)\|_{\mathrm{op}}>s}} n_{\pi}^{2}\|f\|_{L^{p}(\mathbb{G})}\right.
$$


for all $f \in L^{p}(\mathbb{G})$, in view of $\frac{1}{q^{\prime}}-\frac{1}{p^{\prime}}=\frac{1}{p}-\frac{1}{q}=\frac{1}{r}$. Thus, for $1<p \leq 2 \leq q<\infty$, we obtain

$$
\|A f\|_{L^{q}(\mathbb{G})} \lesssim\left(\sup _{s>0} \sum_{\substack{\pi \in \widehat{\mathbb{G}}^{r}>s \\\|\sigma(\pi)\|_{\mathrm{op}}^{r}>s}} n_{\pi}^{2}\|f\|_{L^{p}(\mathbb{G})} .\right.
$$

Further, it can be easily checked that

$$
\begin{aligned}
& \left(\sup _{s>0} \sum_{\substack{\pi \in \widehat{\mathbb{G}} \\
\|\sigma(\pi)\|_{\mathrm{op}}^{r}>s}} n_{\pi}^{2}\right)^{\frac{1}{r}}=\left(\sup _{s>0} \sum_{\substack{\pi \in \widehat{\mathbb{G}} \\
\left\|\sigma_{A}(\pi)\right\|_{\mathrm{op}}>s^{\frac{1}{r}}}} n_{\pi}^{2}\right. \\
& =\left(\sup _{s>0} s^{r} \sum_{\substack{\pi \in \mathbb{G}^{\prime} \\
\left\|\sigma_{A}(\pi)\right\|_{\mathrm{op}}>s}} n_{\pi}^{2}\right)^{\frac{1}{r}}=\sup _{s>0}\left(\sum_{\substack{\pi \in \widehat{\mathbb{G}} \\
\left\|\sigma_{A}(\pi)\right\|_{\mathrm{op}}>s}} n_{\pi}^{2}\right)^{\frac{1}{r}},
\end{aligned}
$$

where in the last equality we used the continuity of the $s^{\frac{1}{r}}$.

\section{Hardy-Littlewood Inequality and Spectral Triples}

As a corollary of Theorem 3.1, we obtain a formal compact quantum group version of the Hardy-Littlewood inequality by using a suitable sequence $\left\{\lambda_{\pi}\right\}$. This is formal in the sense that we do not study underlying inherent geometric data, but nevertheless by formulating this data in terms of an operator $\mathcal{D}$ our quantum Hardy-Littlewood type inequality (5.2) presents in a manner similar to the compact Lie group inequality (1.3).

Theorem 5.1. Let $1<p \leq 2$ and let $\mathbb{G}$ be a compact quantum group of Kac type. Assume that a sequence $\left\{\lambda_{\pi}\right\}_{\pi \in \widehat{\mathbb{G}}}$ grows sufficiently fast, that is,

$$
\sum_{\pi \in \widehat{\mathbb{G}}} \frac{n_{\pi}^{2}}{\left|\lambda_{\pi}\right|^{\beta}}<\infty
$$

Then we have

$$
\sum_{\pi \in \widehat{\mathbb{G}}} n_{\pi}^{2}\left|\lambda_{\pi}\right|^{\beta(p-2)}\left(\frac{\|\widehat{f}(\pi)\|_{\mathrm{HS}}}{\sqrt{n_{\pi}}}\right)^{p} \lesssim\|f\|_{L^{p}(\mathbb{G})}^{p} .
$$

In terms of an unbounded $\mathcal{D}: L^{2}(\mathbb{G}) \rightarrow L^{2}(\mathbb{G})$ defined by $\mathcal{D} \pi=\lambda_{\pi} \pi$, (5.1) and (5.2) are respectively equivalent to $|D|^{-\beta}$ trace class and

$$
\left\|\mathscr{F}_{\mathbb{G}}|\mathcal{D}|^{\beta\left(\frac{1}{2}-\frac{1}{p}\right)} f\right\|_{\ell^{p}(\widehat{\mathbb{G}})} \leq C_{p}\|f\|_{L^{p}(\mathbb{G})} .
$$


Proof of Theorem 5.1. By the construction

$$
C:=\sum_{\pi \in \widehat{\mathbb{G}}} \frac{n_{\pi}^{2}}{\left|\lambda_{\pi}\right|^{\beta}}<+\infty .
$$

Then we have

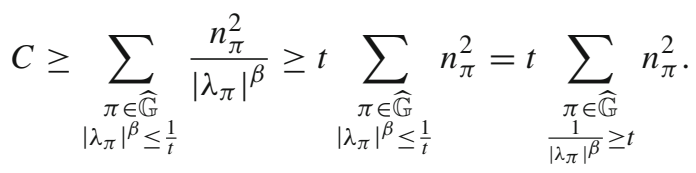

Then by Theorem 3.1, we get (5.2). For the second part, we equivalently view $\left\{\lambda_{\pi}\right\}$ as defining an operator $\mathcal{D}$ and in the case of Kac type, summability with power $\beta$ reduces to condition (5.1). Clearly,

$$
|\mathcal{D}|^{\beta\left(\frac{1}{2}-\frac{1}{p}\right)} \pi_{i j}=\left|\lambda_{\pi}\right|^{\beta\left(\frac{1}{2}-\frac{1}{p}\right)} \pi_{i j} .
$$

Using this and the right-hand side in inequality (5.2), we obtain (5.3).

Although the operator $\mathcal{D}$ is not immediately geometric, it can fit into Connes noncommutative geometric framework of a 'spectral triple'.

Definition 5.2. A spectral triple $(\mathcal{A}, \mathcal{H}, \mathcal{D})$ is a triple consisting of an associative $*-$ subalgebra $\mathcal{A}$ of the algebra $B(\mathcal{H})$ of bounded operators in a separable Hilbert space $\mathcal{H}$ and a linear unbounded self-adjoint operator $\mathcal{D}: \mathcal{H} \rightarrow \mathcal{H}$ with compact resolvent and such that such that

$$
\mathcal{A} \ni a \mapsto \partial(a):=[\mathcal{D}, a] \in B(\mathcal{H}) .
$$

A spectral triple $(\mathcal{A}, \mathcal{H}, \mathcal{D})$ is called summable if $|\mathcal{D}|^{-\beta}$ is trace-class for some $\beta \geq 0$. The infimum of $\beta \in \mathbb{R}_{+}$such that $|\mathcal{D}|^{-\beta+\varepsilon}$ is trace-class for every $\varepsilon>0$ is called the spectral dimension [Con96].

Definition 5.2 is very minimal in the sense that we do not impose any conditions on reality and chirality operators and their interrelations with $\mathcal{D}$ as in [Con95]. It is certainly possible for $\mathcal{D}$ in Theorem 5.1 to obey the further boundedness condition for a spectral triple in the Kac type case, a topic to be explored further elsewhere.

For now, we turn to an explicit construction of spectral triples more relevant to $q$ deformed examples. If $\pi^{k}, \pi^{s} \in \widehat{\mathbb{G}}$ (with labels as shown) then the tensor product $\pi^{k} \otimes \pi^{s}$ is a completely reducible finite-dimensional representation. The matrix elements of $\pi^{k} \otimes \pi^{s}$ are given by

$$
\pi^{k} \otimes \pi^{s}=\left[\pi_{i j}^{k} \pi_{p r}^{s}\right]_{i, j=1, p, r=1}^{n_{k}, n_{s}} .
$$

We shall define the coefficients $C_{i j p r u t}^{k s w}$ as follows

$$
C_{i j p r u t}^{k s w}=\left(\pi_{i j}^{k} \pi_{p r}^{s}, \pi_{u t}^{w}\right)_{L^{2}(\mathbb{G})} .
$$

It then follows from (5.6)

$$
\pi_{i j}^{k} \pi_{p r}^{s}=\sum_{w \in I_{k s}} \sum_{u, t=1}^{n_{m}} C_{i j p r u t}^{k s w} \pi_{u t}^{w},
$$


where $I_{k s}$ is a finite subset of $\mathbb{N}$. These Clebsch-Gordan coefficients are important to write down the action of the commutator $\partial(a)=[\mathcal{D}, a]$ explicitly. In [CP08], these coefficients were computed for the quantum groups $\mathrm{SU}_{2 l+1}^{q}$, allowing the authors compute the action of the left multiplication operator on $L^{2}\left(\mathrm{SU}_{2 l+1}^{q}\right)$ and leading in turn to growth restriction on the eigenvalues $\lambda_{k}$. In order to consider this more generally, we take a slightly different approach and leave the Clebsch-Gordan coefficients in the bound as they depend on the compact quantum group.

It is convenient, however, to focus on the compact matrix quantum group case [Wor87, MVD98]. Here there is a matrix of generators $u_{m n}$ of $\mathbb{C}[\mathbb{G}]$ corresponding to a defining unitary representation. We can expand them as

$$
u_{m n}=\sum_{k} \sum_{i j} \alpha_{m n}^{i j k} \pi_{i j}^{k} .
$$

It can be clearly seen from the Peter-Weyl decomposition that there are only finitely many non-zero $\alpha_{m n}^{i j k}$ for each generator $u_{m n}$. These coefficients are closely related to the Clebsch-Gordan coefficients.

Theorem 5.3. Let $(\mathbb{G}, \Delta)$ be a compact matrix quantum group and $\mathcal{D}: L^{2}(\mathbb{G}) \rightarrow L^{2}(\mathbb{G})$ the unbounded linear operator given by $\mathcal{D} \pi_{i j}^{k}=\lambda_{k} \pi_{i j}^{k}$. Then $\left(\mathbb{G}, L^{2}(\mathbb{G}), \mathcal{D}\right)$ is a spectral triple if and only if

$$
\sqrt{\sum_{i, j, k}\left|\lambda_{k}-\lambda_{s}\right|^{2} \sum_{w \in I_{k s}} \sum_{t=1}^{n_{w}}\left|\alpha_{m n}^{i j k} C_{i j p r t t}^{k s w}\right|^{2} \frac{q_{t}^{w}}{d_{\pi^{w}}}} \leq C \sqrt{\frac{q_{r}^{s}}{d_{\pi^{s}}}}
$$

holds for all $s, m, n, r$ and some constant $C$.

Example 5.4 (Equivariant spectral triples on the quantum $\mathrm{SU}_{n}^{q}$ ). Condition (5.9) imposes certain growth condition on consecutive differences $\left|\lambda_{k}-\lambda_{s(k)}\right|$ of the eigenvalues $\lambda_{k}$. For $\mathbb{G}=\mathrm{SU}_{n}^{q}$, it is possible to compute [CP08] the coefficients $C_{i j p r u t}^{k s m}$. In more detail, the authors showed that the $C_{i j p r t t}^{k s m}$ 's are essentially powers of $q$, i.e.

$$
C_{i j p r t t}^{k s m}=q^{C^{\prime}}
$$

where the exponent $C^{\prime}=C^{\prime}(k, s, m, i, k, p, r, t)$ is determined by $k, s, m$. For more details we refer to [CP08, pp. 30-32].

Proof of Theorem 5.3. The generators $u_{m n}$ are dense in $\mathbb{C}[\mathbb{G}]$. Therefore we concentrate on showing that the commutator

$$
\partial(a): \mathcal{H} \ni b \mapsto \partial(a) b=[\mathcal{D}, a] b \in \mathcal{H}
$$

is bounded on $u_{m n}$ if and only if condition (5.9) is true. Let $u_{m n} \in \mathbb{C}[\mathbb{G}], \pi^{s} \in \widehat{\mathbb{G}}$ and take $a=u_{m n}$ and $b=\pi_{p r}^{s}$. The action of $\mathcal{D}$ on $u_{m n}$ is as follows

$$
\mathcal{D} u_{m n}=\sum_{k} \lambda_{k} \sum_{i j} \alpha_{m n}^{i j k} \pi_{i j}^{k}
$$

Assume that $\partial(a)=[\mathcal{D}, \cdot]$ is bounded for all $a \in \mathbb{C}[\mathbb{G}]$, i.e.

$$
\|\partial(a) b\|_{L^{2}(\mathbb{G})} \leq C_{a}\|b\|_{L^{2}(\mathbb{G})}, \quad b \in \mathbb{C}[\mathbb{G}] .
$$


In particular, for $a=u_{m n} \in \mathbb{C}[\mathbb{G}]$ and $b=\pi_{p r}^{s} \in \mathbb{C}[\mathbb{G}]$, we get

$$
\left\|\partial\left(u_{m n}\right) \pi_{p r}^{s}\right\|_{L^{2}(\mathbb{G})} \leq C_{a}\left\|\pi_{p r}^{s}\right\|_{L^{2}(\mathbb{G})} .
$$

Then by the direct computation

$$
\begin{aligned}
\left\|\partial\left(u_{m n}\right) \pi_{p r}^{s}\right\|_{L^{2}(\mathbb{G})}^{2} & =\left\|\left(\mathcal{D} u_{m n}\right) \pi_{p r}^{s}-u_{i j}\left(\mathcal{D} \pi_{p r}^{s}\right)\right\|_{L^{2}(\mathbb{G})}^{2} \\
& =\left\|\left(\sum_{k} \lambda_{k} \sum_{i j} \alpha_{m n}^{i j k} \pi_{i j}^{k}\right) \pi_{p r}^{s}-\lambda_{s} \sum_{k} \sum_{i j} \alpha_{m n}^{i j k} \pi_{i j}^{k} \pi_{p r}^{s}\right\|_{L^{2}(\mathbb{G})}^{2} \\
& =\sum_{i, j, k}\left|\lambda_{k}-\lambda_{s}\right|^{2}\left\|\pi_{i j}^{k} \pi_{p r}^{s}\right\|_{L^{2}(\mathbb{G})}^{2} \\
& =\sum_{i, j, k}\left|\lambda_{k}-\lambda_{s}\right|^{2} \sum_{w \in I_{k s}} \sum_{t=1}^{n_{w}}\left|\alpha_{m n}^{i j k} C_{i j p q t t}^{k s w}\right|^{2} \frac{q_{t}^{w}}{d_{\pi}^{w}} .
\end{aligned}
$$

Using that

$$
\left\|\pi_{p r}^{s}\right\|_{L^{2}(\mathbb{G})}^{2}=\frac{q_{r}^{s}}{d_{\pi^{r}}}
$$

and (5.15), we obtain (5.9). The converse is also true. Indeed, writing inequality (5.15) in the reverse order, we get (5.14).

Example 5.5. Let $\mathbb{G}=C(G)$ where $G$ is a compact Lie group. One can take $\mathcal{D}=$ $\sqrt{1-\Delta_{G}}$ where $\Delta_{G}$ is the Laplacian on $G$.

Example 5.6 ([CP08]). Let $\mathbb{G}=\mathrm{SU}_{2}^{\mathrm{q}}$ and $L^{2}\left(\mathrm{SU}_{2}^{\mathrm{q}}\right)$ be the GNS-space. Let $\mathcal{D}$ be a Dirac operator operator acting on the entries $t_{i j}^{l}$ of the irreducible corepresentations $t^{l} \in \widehat{\mathrm{SU}_{2}^{\mathrm{q}}}$ of $\mathrm{SU}_{2}^{\mathrm{q}}$ as follows

$$
\mathcal{D} t_{i j}^{l}= \pm(2 l+1) t_{i j}^{l}, \quad i, j=0, \frac{1}{2}, \ldots, l, l \in \frac{1}{2} \mathbb{N}_{0} .
$$

In this example, we have $C_{\mathcal{D}}^{\infty}=\mathrm{SU}_{2}^{\mathrm{q}}$. The Chern character corresponding to $\left(\mathrm{SU}_{2}^{\mathrm{q}}\right.$, $\left.L^{2}\left(\mathrm{SU}_{2}^{\mathrm{q}}\right), \mathcal{D}\right)$ is non-trivial [CP08].

We do not consider $\beta$-summability in Theorem 5.3. It is known to hold for some $\beta$ in the case of Example 5.6, see [CP08]. Also note that we cannot immediately apply Theorem 5.3 to $\mathbb{G}=\mathrm{SU}_{2}^{\mathrm{q}}$ with $\lambda_{k}$ given more naturally by $q$-integers as we will do later. Indeed, it has been already observed in [Con08] that standard quantum group examples do not very naturally fit into the classical theory of spectral triples, which may need to be replaced by a twisted version for example in the commutator (5.11). In this context it is also possiblel to demand a 'quantum summability' condition

$$
\sum_{k \in \mathbb{N}} \frac{d_{k}^{2}}{\left|\lambda_{k}\right|^{\beta}}<+\infty
$$

for some $\beta>0$, i.e. with respect to a modified 'quantum' trace. This reduces correctly for classical compact Lie groups and compact quantum groups of Kac type, and also holds for $\mathbb{G}=\mathrm{SU}_{2}^{\mathrm{q}}$ if we use $q$-integers for the $\lambda_{k}$ but not if we use the classical values in Example 5.6. 


\section{Schwartz Kernels}

Definition 6.1 (Smooth domain). Let $\mathbb{G}$ be a compact quantum group and let $\mathcal{D}: \mathbb{C}[\mathbb{G}] \rightarrow$ $\mathbb{C}[\mathbb{G}]$ be a linear map extended to $L^{2}(\mathbb{G}) \rightarrow L^{2}(\mathbb{G})$ as a closed unbounded linear operator. Then the smooth domain $C_{\mathcal{D}}^{\infty} \subset \mathbb{G}$ of $\mathcal{D}$ is defined as follows

$$
C_{\mathcal{D}}^{\infty}:=\bigcap_{\alpha \geq 0} \operatorname{Dom}\left(|\mathcal{D}|^{\alpha}\right)
$$

The Frechet structure is given by the seminorms

$$
\|\varphi\|_{\alpha}=\left\||\mathcal{D}|^{\alpha} \varphi\right\|_{L^{2}(\mathbb{G})}, \quad \varphi \in C_{\mathcal{D}}^{\infty}, \alpha \geq 0 .
$$

The powers $|\mathcal{D}|^{\alpha}$ are defined by the spectral theorem. It can be checked that $C_{\mathcal{D}}^{\infty}$ is a locally convex topological vector space.

We show that every linear operator $A: C_{\mathcal{D}}^{\infty} \rightarrow C_{\mathcal{D}}^{\infty}$ continuous with respect to the Frechet topology can be associated with a distribution $K_{A}$ 'acting' on $\mathbb{G} \times \mathbb{G}$. In other words, every linear continuous operator $A: C_{\mathcal{D}}^{\infty} \rightarrow C_{\mathcal{D}}^{\infty}$ possesses a Schwartz kernel $K_{A}$. This allows us to define the global symbol of $A$ in line with the pseudo-differential calculus on compact Lie groups [RT13, RT10]. The global symbols have been recently studied [LNJP16] on the quantum tori $\mathbb{T}_{\theta}^{n}, n \in \mathbb{N}, \theta \in \mathbb{R}$.

Definition 6.2 (Rapidly decreasing functions on $\widehat{\mathbb{G}})$. Denote by $\mathcal{S}(\widehat{\mathbb{G}})$ the space of matrixvalued sequences $\{\sigma(\pi)\}, \sigma(\pi) \in \mathbb{C}^{n_{\pi} \times n_{\pi}}$ satisfying the conditions

$$
\mathcal{S}(\widehat{\mathbb{G}})=\left\{\sigma=\{\sigma(\pi)\}_{\pi \in \widehat{\mathbb{G}}}: \sum_{\pi \in \widehat{\mathbb{G}}} d_{\pi}\left|\lambda_{\pi}\right|^{2 \alpha}\|\sigma(\pi)\|_{\mathrm{HS}}^{2}<+\infty \text { for any } \alpha \geq 0\right\} .
$$

The space $\mathcal{S}(\widehat{\mathbb{G}})$ becomes a locally convex topological space if we endow it with the norms

$$
p_{\alpha}(\sigma):=\left(\sum_{\pi \in \widehat{\mathbb{G}}} d_{\pi}\left|\lambda_{\pi}\right|^{2 \alpha}\|\sigma(\pi)\|_{\mathrm{H} S}^{2}\right)^{\frac{1}{2}}, \quad \alpha \geq 0 .
$$

The construction of the topology on $C_{\mathcal{D}}^{\infty}(\mathbb{G})$ readily implies that the quantum Fourier transform $\mathscr{F}_{\mathbb{G}}$ is a homeomorphism between $C_{\mathcal{D}}^{\infty}$ and $\mathcal{S}(\widehat{\mathbb{G}})$.

Definition 6.3 (Distributions). Let us denote by $\mathcal{S}^{\prime}(\mathbb{G})$ the space $\left[C_{\mathcal{D}}^{\infty}(\mathbb{G})\right]^{*}$ of all linear functionals continuous with respect to the topology on $C_{\mathcal{D}}^{\infty}(\mathbb{G})$, i.e.

$$
\mathcal{S}^{\prime}(\mathbb{G}):=\left[C_{\mathcal{D}}^{\infty}(\mathbb{G})\right]^{*} .
$$

Let us denote by $\mathcal{S}^{\prime}(\widehat{\mathbb{G}})$ the space $[\mathcal{S}(\widehat{\mathbb{G}})]^{*}$ of all linear linear continuous functionals on $\mathcal{S}(\widehat{\mathbb{G}})$, i.e.

$$
\mathcal{S}^{\prime}(\widehat{\mathbb{G}}):=[\mathcal{S}(\widehat{\mathbb{G}})]^{*} .
$$

Definition 6.4. For any distribution $u \in \mathcal{S}^{\prime}(\widehat{\mathbb{G}})$ its Fourier transform $\widehat{u}$ is a distribution on $C_{\mathcal{D}}^{\infty}(\mathbb{G})$ given by

$$
\widehat{u}(\widehat{f}):=u(f), \quad \widehat{f} \in \mathcal{S}(\widehat{\mathbb{G}}), f \in C_{\mathcal{D}}^{\infty}(\mathbb{G}) .
$$


Proposition 6.5. A linear function u on $C_{\mathcal{D}}^{\infty}$ is a distribution, if and only if, there exists a constant $C$ and $a$ number $\alpha \geq 0$ such that

$$
|u(f)| \leq C\left(\sum_{\pi \in \widehat{\mathbb{G}}} d_{\pi}\left|\lambda_{\pi}\right|^{2 \alpha}\|\widehat{f}(\pi)\|_{\mathrm{HS}}^{2}\right)^{\frac{1}{2}},
$$

for every $f \in C_{\mathcal{D}}^{\infty}(\mathbb{G})$.

Proposition 6.6. The space $\mathcal{S}^{\prime}(\mathbb{G})$ is complete, i.e. for every Cauchy sequence $\left\{u_{n}\right\} \subset$ $\mathcal{S}^{\prime}(\mathbb{G})$ the limit

$$
u=\lim u_{n} \in \mathcal{S}^{\prime}(\mathbb{G})
$$

exists and belongs to $\mathcal{S}^{\prime}(\mathbb{G})$. If $\varphi_{n}$ converges to $\varphi$ in $C_{\mathcal{D}}^{\infty}$, then

$$
\lim _{n} u_{n}\left(\varphi_{n}\right)=u(\varphi) \text {. }
$$

Both of these are by standard methods and hold in the general situation[Tre67]. By transposing the inverse Fourier transform $\mathscr{F}_{\mathbb{G}}^{-1}: \mathcal{S}\left(\widehat{\mathbb{G}}^{-}\right) \rightarrow C_{\mathcal{D}}^{\infty}(\mathbb{G})$, the Fourier transform $\mathscr{F}_{\mathbb{G}}$ extends uniquely to a mapping

$$
\mathscr{F}_{\mathbb{G}}: \mathcal{S}^{\prime}(\mathbb{G}) \rightarrow \mathcal{S}^{\prime}(\widehat{\mathbb{G}})
$$

by the formula

$$
\mathscr{F}_{\mathbb{G}}[u](\sigma)=u\left(\mathscr{F}_{\mathbb{G}}^{-1}[\sigma]\right), \quad u \in \mathcal{S}^{\prime}(\mathbb{G}) .
$$

In other words, for every distribution $u \in \mathcal{S}^{\prime}(\mathbb{G})$ its Fourier transform $\mathscr{F}_{\mathbb{G}}[u]$ is a distribution on $\mathcal{S}(\widehat{\mathbb{G}})$.

Definition 6.7. For any distribution $u \in \mathcal{S}^{\prime}(\mathbb{G})$ its Fourier transform $\widehat{u}$ is a distribution on $\mathcal{S}^{\prime}(\widehat{\mathbb{G}})$ given by

$$
\widehat{u}(\sigma):=u\left(\mathscr{F}_{\mathbb{G}}^{-1}(\sigma)\right), \quad \sigma \in \mathcal{S}(\widehat{\mathbb{G}}) .
$$

Proposition 6.8. Let $(\mathbb{G}, \Delta)$ be a compact quantum group and let $|\mathcal{D}|^{-\beta}$ be of trace class for some $\beta>0$. Then the Frechet space $C_{\mathcal{D}}^{\infty}$ is nuclear.

Proof of Proposition 6.8. It is sufficient to prove that $\mathcal{S}(\widehat{\mathbb{G}})$ is a nuclear Frechet space since $\mathscr{F}_{\mathbb{G}}$ is a homeomorphism. The former fact follows from [Tri78, Section 8.2.1].

The theory of topological vector spaces has been significantly developed [Gro55] by Alexander Grothendieck. It turns out that the property of being nuclear is crucial and these spaces are 'closest' to finite-dimensional spaces. The nuclearity is the necessary and sufficient condition for the existence of abstract Schwartz kernels.

The topological tensor product preserves nuclearity [Tre67].

Definition 6.9. A linear continuous operator $A: C_{\mathcal{D}}^{\infty}(\mathbb{G}) \mapsto \mathcal{S}^{\prime}(\mathbb{G})$ is called a pseudodifferential operator.

From the abstract Schwartz kernel theorem [Tre67], we readily obtain

Theorem 6.10. Let $\mathcal{D}$ be as in Proposition 6.8. Let $A: C_{\mathcal{D}}^{\infty} \rightarrow \mathcal{S}^{\prime}(\mathbb{G})$ be a pseudodifferential operator. Then there is a distribution $K_{A} \in \mathcal{S}^{\prime}(\mathbb{G}) \widehat{\otimes} \mathcal{S}^{\prime}(\mathbb{G})$ such that

$$
C_{\mathcal{D}}^{\infty} \ni \varphi \mapsto A \varphi \in \mathcal{S}^{\prime}(\mathbb{G}), \quad \varphi \rightarrow(A \varphi)(\psi)=K_{A}(\varphi \otimes \psi), \quad \psi \in C_{\mathcal{D}}^{\infty} .
$$


The structure theorem [Tre67, Theorem 45.1] applied to the topological tensor product $\mathcal{S}^{\prime}(\mathbb{G}) \widehat{\otimes} \mathcal{S}^{\prime}(\mathbb{G})$ immediately yields that the Schwartz kernel $K_{A}$ can be written in the form

$$
K_{A}=\sum_{n=1}^{\infty} s_{n}^{A} x_{n}^{A} \otimes t_{n}^{A},
$$

where $\sum_{n=1}^{\infty}\left|s_{n}^{A}\right|<+\infty$ and $\left\{x_{n}\right\},\left\{t_{n}\right\} \subset \mathcal{S}^{\prime}(\mathbb{G})$ tend to 0 in $\mathcal{S}^{\prime}(\mathbb{G})$. This allows us to define global symbols $\sigma_{A}$ in line with the classical theory.

Definition 6.11. Let $A: C_{\mathcal{D}}^{\infty}(\mathbb{G}) \rightarrow \mathcal{S}^{\prime}(\mathbb{G})$ be a pseudo-differential operator. We define a global symbol $\sigma_{A}$ of $A$ at $\pi \in \widehat{\mathbb{G}}$ as a matrix $\sigma_{A}(\pi)=\left[\sigma_{A}\left(\pi_{i j}\right)\right]_{i j}^{n_{\pi}}$ of distributions $\sigma_{A}\left(\pi_{i j}\right) \in \mathcal{S}^{\prime}(\mathbb{G})$ acting by the formula

$$
C_{\mathcal{D}}^{\infty}(\mathbb{G}) \ni \varphi \mapsto \sigma_{A}\left(\pi_{i j}\right)(\varphi):=K_{A}\left(\varphi \otimes \pi_{i j}\right) \in \mathbb{C} .
$$

Alternatively, we have

$$
\sigma_{A}(\pi)_{i j}=\sum_{n=1}^{\infty} s_{n}^{A} x_{n}^{A} h\left(t_{n}^{A} \pi_{j i}^{*}\right) \in \mathcal{S}^{\prime}(\mathbb{G}) .
$$

Definition 6.12. We say that a pseudo-differential operator $A: C_{\mathcal{D}}^{\infty}(\mathbb{G}) \rightarrow \mathcal{S}^{\prime}(\mathbb{G})$ is regular if $K_{A} \in C_{\mathcal{D}}^{\infty} \widehat{\otimes} C_{\mathcal{D}}^{\infty}$.

It can be easily seen that this class of pseudo-differential operators is closed under composition. Explicit composition formula for the global symbols on quatum tori $\mathbb{T}_{\theta}^{n}$ has been recently obtained in [LNJP16]. In Theorem 6.13 we derive the composition formula in terms of abstract Schwartz kernels.

Theorem 6.13. Let $A: C_{\mathcal{D}}^{\infty} \rightarrow C_{\mathcal{D}}^{\infty}$ and $B: C_{\mathcal{D}}^{\infty} \rightarrow C_{\mathcal{D}}^{\infty}$ be two regular pseudodifferential operators on $\mathbb{G}$. Let $K_{A}$ and $K_{B}$ be the Schwartz kernels of $A$ and $B$. Then the composition $A \circ B: C_{\mathcal{D}}^{\infty} \rightarrow C_{\mathcal{D}}^{\infty}$ is a regular pseudo-differential operator. Moreover, the Schwartz kernel $K_{A B}$ of the composition $A B$ is given by

$$
K_{A B}=\sum_{n} s_{n}^{A}\left(\sum_{m} s_{m}^{B} x_{n}^{A}\left(x_{m}^{B}\right) t_{m}^{B}\right) \otimes t_{n}^{A},
$$

where $K_{A}=\sum_{n} s_{n}^{A} x_{n}^{A} \otimes t_{n}^{A}$ and $K_{B}=\sum_{m} s_{m}^{B} x_{m}^{B} \otimes t_{m}^{B}$.

Proof of Theorem 6.13. Our proof relies on the theory of topological vector spaces. We notice that

$$
B f=\sum_{m} s_{m}^{B} x_{m}^{B} t_{m}^{B}(f), \quad x_{m}^{B}, f \in C_{\mathcal{D}}^{\infty} .
$$

By explicit calculations, we have

$$
\begin{aligned}
(A B f, g)_{L^{2}(\mathbb{G})}=( & (B f), g)_{L^{2}(\mathbb{G})}=\sum_{n} s_{n}^{A} x_{n}^{A}(B f) t_{n}^{A}(g) \\
& =\sum_{n} s_{n}^{A} x_{n}^{A}\left(\sum_{m} s_{m}^{B} x_{m}^{B} t_{m}^{B}(f)\right) t_{n}^{A}(g) \\
& =\sum_{n} \sum_{m} s_{n}^{A} s_{m}^{B} x_{n}^{A}\left(x_{m}^{B}\right) t_{m}^{B}(f) t_{n}^{A}(g),
\end{aligned}
$$


where in the second equality we used that $\sum_{m}^{N} s_{m}^{B} x_{m}^{B} t_{m}^{B}(f) \in C_{\mathcal{D}}^{\infty}$ converges to $\sum_{m}^{\infty}$ $s_{m}^{B} x_{m}^{B} t_{m}^{B}(f) \in C_{\mathcal{D}}^{\infty}$ with respect to the topology in $C_{\mathcal{D}}^{\infty}$. This shows (6.7).

Now, we show that $K_{A B} \in C_{\mathcal{D}}^{\infty} \widehat{\otimes} C_{\mathcal{D}}^{\infty}$. Denoting

$$
\begin{aligned}
x_{n}^{A B} & =\sum_{m} s_{m}^{B} x_{n}^{A}\left(x_{m}^{B}\right) t_{m}^{B}, \\
t_{n}^{A B} & =t_{n}^{A}, \\
s_{n}^{A B} & =s_{n}^{A},
\end{aligned}
$$

we can write the Schwartz kernel $K_{A B}$ as follows

$$
K_{A B}=\sum_{n} s_{n}^{A B} x_{n}^{A B} \otimes t_{n}^{A B} .
$$

Since $A$ is a regular pseudo-differential operator, we have

$$
\sum_{n}\left|s_{n}^{A B}\right|<\infty
$$

and $t_{n}^{A B} \in C_{\mathcal{D}}^{\infty}$ and $t_{n}^{A B} \rightarrow 0$. Hence, it only remains to check that $x_{n}^{A B} \in C_{\mathcal{D}}^{\infty}$ and $x_{n}^{A B} \rightarrow 0$, i.e. for every $\alpha>0$

$$
p_{\alpha}\left(x_{n}^{A B}\right) \rightarrow 0, \quad n \rightarrow \infty .
$$

Since the sequence $\left\{s_{m}^{B} x_{n}^{A}\left(x_{m}^{B}\right) t_{m}^{B}\right\}_{m=1}^{\infty} \subset C_{\mathcal{D}}^{\infty}$ is summable, we get

$$
p_{\alpha}\left(\sum_{m=1}^{\infty} s_{m}^{B} x_{n}^{A}\left(x_{m}^{B}\right) t_{m}^{B}\right) \leq \sum_{m=1}^{\infty}\left|s_{m}^{B} x_{n}^{A}\left(x_{m}^{B}\right)\right| p_{\alpha}\left(t_{m}^{B}\right) \leq C \sum_{m=1}^{\infty}\left|s_{m}^{B}\right| \mid x_{n}^{A}\left(x_{m}^{B}\right) .
$$

The injective topology on $\mathcal{S}^{\prime}(\mathbb{G})$ yields that $x_{n}^{A}$ tends to 0 uniformly over bounded subsets of $C_{\mathcal{D}}^{\infty}$, i.e.

$$
\lim _{n} x_{n}^{A}\left(x_{m}^{B}\right)=0 .
$$

Thus, using this and passing to the limit in (6.10), we obtain (6.9). We refer to [Tre67, Part III] for further details and definitions.

We introduce the right-convolution Schwartz kernel $R_{A}$ by the formula

$$
R_{A}:=\sum_{n=0}^{\infty} s_{n}^{A} x_{n}^{A} \otimes u_{n}^{A},
$$

where $u_{n}^{A}$ is a convolution type vector-valued distribution acting by the formula

$$
C_{\mathcal{D}}^{\infty} \ni \varphi \rightarrow u_{n}^{A}(\varphi)=(1 \otimes h)\left(\left(1 \otimes u_{n}^{A}\right)(1 \otimes S) \Delta \varphi\right) \in C_{\mathcal{D}}^{\infty},
$$

and $s_{n}^{A}$ are as in (6.4). 
Theorem 6.14. Let $(\mathbb{G}, \Delta)$ be a compact quantum group and let $A: C_{\mathcal{D}}^{\infty} \rightarrow C_{\mathcal{D}}^{\infty}$ be a regular pseudo-differential operator acting via right-convolution kernel, i.e.

$$
C_{\mathcal{D}}^{\infty}(\mathbb{G}) \ni f \mapsto A f=\sum_{n=0}^{\infty} s_{n}^{A} x_{n}^{A} u_{n}^{A}(f) \in C_{\mathcal{D}}^{\infty}(\mathbb{G}) .
$$

Then we have

$$
A f=\sum_{\pi \in \widehat{\mathbb{G}}} d_{\pi} \operatorname{Tr}\left(\sigma_{A}(\pi) \widehat{f}(\pi) \pi\right),
$$

where $\sigma_{A}(\pi)$ is the global symbol of A defined by (6.5).

Proof of Theorem 6.14. Let $f \in \mathbb{C}[\mathbb{G}]$. Then we have

$$
f=\sum_{\pi \in I_{f}} d_{\pi} \sum_{i, j=1}^{n_{\pi}} \widehat{f}(\pi)_{i j} \pi_{j i}
$$

We shall start by showing that (6.12) holds true for $f \in \mathbb{C}[\mathbb{G}]$. We have

$$
\begin{aligned}
A f & =\sum_{n=0}^{\infty} s_{n}^{A} x_{n}^{A} \otimes u_{n}^{A}(f)=\sum_{n=0}^{\infty} s_{n}^{A} x_{n}^{A} \otimes(1 \otimes h)\left(u_{n}^{A} \Delta f\right) \\
& =\sum_{n=0}^{\infty} s_{n}^{A} x_{n}^{A} \otimes(1 \otimes h)\left(\left(1 \otimes u_{n}^{A}\right) \cdot \sum_{\pi \in I_{f}} d_{\pi} \sum_{i, j=1}^{n_{\pi}} \widehat{f}(\pi)_{i j}(1 \otimes S) \Delta \pi_{j i}\right) \\
& =\sum_{\pi \in I_{f}} \sum_{i, j=1}^{n_{\pi}} \sum_{k=1}^{n_{\pi}} \sum_{n=0}^{\infty} s_{n}^{A} x_{n}^{A} \otimes(1 \otimes h)\left[d_{\pi} \widehat{f}(\pi)_{i j} \pi{ }_{j k} \otimes u_{n}^{A} \pi_{k i}^{*}\right] \\
& =\sum_{\pi \in I_{f}} d_{\pi} \sum_{i, j=1}^{n_{\pi}} \sum_{k=1}^{n_{\pi}} \widehat{f}(\pi)_{i j} \pi_{j k} \cdot \sum_{n=0}^{\infty} s_{n}^{A} x_{n}^{A} h\left(u_{n}^{A} \pi_{k i}^{*}\right) \\
& =\sum_{\pi \in I_{f}} d_{\pi} \sum_{i, j=1}^{n_{\pi}} \sum_{k=1}^{n_{\pi}} u_{n}^{A} \widehat{f}(\pi)_{i j} \pi_{j k} \cdot \sum_{n=0}^{\infty} s_{n}^{A} x_{n}^{A} h\left(u_{n}^{A} \pi_{k i}^{*}\right) \\
& =\sum_{\pi \in I_{f}} d_{\pi} \operatorname{Tr}\left[\widehat{f}(\pi) \pi \sigma_{A}(\pi)\right] .
\end{aligned}
$$

Therefore, we get (6.12) where we can replace $I_{f}$ by $\widehat{\mathbb{G}}$.

\section{Differential Calculi on Compact Quantum Groups}

In this section we are going to ask how the above 'Fourier approach' to the analysis on compact quantum groups interplays with the theory of differential structures on Hopf algebras of compact quantum groups and how this extends to $C_{\mathcal{D}}^{\infty}(\mathbb{G})$. Recall that differential structures in the literature have been defined at the polynomial level i.e. on $\mathbb{C}[\mathbb{G}]$ as a Hopf $*$-algebra. For every choice of $\left\{\lambda_{\pi}\right\}_{\pi \in \hat{\mathbb{G}}}$ we have an operator defined by $\mathcal{D} \pi=\lambda_{\pi} \pi$ and

$$
\mathbb{C}[\mathbb{G}] \subseteq C_{\mathcal{D}}^{\infty}(\mathbb{G}) \subseteq \mathbb{G}
$$


Thus for $\mathbb{G}=\mathrm{SU}_{2}^{\mathrm{q}}$ we have $\mathbb{C}[\mathbb{G}]=\mathbb{C}_{q}\left[S U_{2}\right]$ as the usual dense Hopf $*$-subalgebra of $\mathrm{SU}_{2}^{\mathrm{q}}$ with a $2 \times 2$ matrix of generators while $C_{\mathcal{D}}^{\infty}\left(\mathrm{SU}_{2}^{\mathrm{q}}\right)$ lies in between as something more akin to $C^{\infty}\left(S U_{2}\right)$. Our goal in the present section is to show that elements of $C_{\mathcal{D}}^{\infty}(\mathbb{G})$ are indeed smooth with respect to a suitable differential structure at least for $\mathrm{SU}_{2}^{\mathrm{q}}$ and in outline for the general $q$-deformation case.

We start by recalling the purely algebraic definition of first-order differential calculus over associative algebras and refer to [Maj16] for a thorough exposition. Let $A$ be a unital algebra over a field $k$.

Definition 7.1. A first order differential calculus $\left(\Omega^{1}, \mathrm{~d}\right)$ over $A$ means

(1) $\Omega^{1}$ is an $A$-bimodule.

(2) $\mathrm{d}: A \rightarrow \Omega^{1}$ is a linear map satisfying

$$
\mathrm{d}(a b)=(\mathrm{d} a) b+a \mathrm{~d} b, \quad \forall a, b \in A .
$$

(3) The vector space $\Omega^{1}$ is spanned by elements of the form $a \mathrm{~d} b$,

$$
\Omega^{1}=\operatorname{span}\{a \mathrm{~d} b\}_{a, b \in A} .
$$
d.

In the $*$-algebra case, $*$ extends uniquely to $\Omega^{1}$ in such a way that it commutes with

Example 7.2. Let $A=C^{\infty}(\mathbb{R})$ and $\Omega^{1}=C^{\infty}(\mathbb{R})$.d $x$ with left and right action given by multiplication in $C^{\infty}(\mathbb{R})$ (so functions and $\mathrm{d} x$ commute). The exterior derivative is $\mathrm{d} f=\frac{\partial f}{\partial x} \mathrm{~d} x$ as this is the classical calculus.

There are many other interesting calculi even on the commutative algebra of functions in one variable, see [Maj16].

Definition 7.3. A differential calculus $\left(\Omega^{1}, \mathrm{~d}\right)$ over a Hopf algebra $A$ is called leftcovariant if

(1) There is a left coaction $\Delta_{L}: \Omega^{1} \rightarrow A \otimes \Omega^{1}$.

(2) $\Omega^{1}$ with its given left action becomes a left Hopf module in the sense $\Delta_{L}(a \omega)=$ $(\Delta a) .\left(\Delta_{L} \omega\right)$ for all $a \in A, \omega \in \Omega^{1}$.

(3) The exterior derivative $\mathrm{d}: A \rightarrow \Omega^{1}$ is a comodule map, where $A$ coacts on itself by the coproduct $\Delta$

This case was first analysed in [Wor89] but here we continue with a modern algebraic exposition. Note that the last two requirements imply that $\Delta_{L}(a \mathrm{~d} b)=$ $a_{(1)} b_{(1)} \otimes a_{(2)} \mathrm{d} b_{(2)}$ using the Sweedler notation $\Delta a=a_{(1)} \otimes a_{(2)}$, and conversely if this formula gives a well-defined map then one can show that it makes the calculus left covariant. Hence this is a property of $\left(\Omega^{1}, \mathrm{~d}\right)$ not additional data. We have a similar notion of right covariance and the calculus is called bicovariant if it is both left and right covariant. Let $\Lambda^{1}=\left\{\omega \in \Omega^{1} \mid \Delta_{L} \omega=1 \otimes \omega\right\}$ be the space of left-invariant 1-forms on a left-covariant calculus.

In this case we define the Maurer-Cartan form $\varpi: A^{+} \rightarrow \Lambda^{1}$ by

$$
\varpi(a)=S a_{(1)} \mathrm{d} a_{(2)}
$$

. This map is surjective by the spanning assumption above and is a right $A$-module map, since

$$
\varpi(a b)=\left(S b_{(1)}\right)\left(S a_{(1)}\right)\left(\mathrm{d} a_{(2)}\right) b_{(2)}+\left(S b_{(1)}\right) \varepsilon(a) \mathrm{d} b_{(2)}=\varpi(a) \triangleleft b,
$$


where $\Lambda^{1}$ is a right module by $\omega \triangleleft b=\left(S b_{(1)}\right) \omega b_{(2)}$. Hence $\Lambda^{1} \cong A^{+} / I$ for some right ideal $I \subset A^{+}$. Conversely, given a right $A$-module $\Lambda^{1}$ and a surjective right-module map $\varpi: A^{+} \rightarrow \Lambda^{1}$ we can define a left covariant calculus by exterior derivative and bimodule relations

$$
\mathrm{d} a=a_{(1)} \varpi \pi_{\varepsilon} a_{(2)}, \quad \omega a=a_{(1)}\left(\omega \triangleleft a_{(2)}\right), \quad \forall a \in A, \omega \in \Lambda^{1} .
$$

Here $\pi_{\varepsilon}(a)=a-\varepsilon(a)$ projects $A \rightarrow A^{+}$and $\Omega^{1}=A . \Lambda^{1}$ is free as a left module. If the calculus is bicovariant then $\Lambda^{1}$ also has a right coaction making $\Lambda^{1}$ an object in the braided category $\mathcal{M}_{A}^{A}$ of crossed $A$-modules (also called Radford-Drinfeld-Yetter modules). Here $A^{+}$for any Hopf algebra is also a right crossed module by

$$
a \triangleleft b=a b, \quad \operatorname{Ad}_{R}(a)=a \otimes\left(S a_{(1)}\right) a_{(3)}
$$

(the right adjoint coaction) and $\varpi: A^{+} \rightarrow \Lambda^{1}$ becomes a surjective morphism of right crossed-modules in the bicovariant case.

Now let the calculus be bicovariant and $\Lambda^{1}$ of finite dimension $n$ as a vector space ( $\Omega^{1}$ finite-dimensional over $A$ ) and $\left\{e_{i}\right\}_{i=1}^{n}$ a basis of $\Lambda^{1}$ with $\left\{f^{i}\right\}$ a dual basis. Then the associated 'left-invariant vector fields' (which are not necessarily derivations) are given by

$$
\partial^{i}: A \rightarrow A, \quad \partial^{i} a=a_{(1)} f^{i}\left(\varpi \pi_{\varepsilon} a_{(2)}\right), \quad \forall a \in A
$$

and obey $\Delta \partial^{i}=\left(\mathrm{id} \otimes \partial^{i}\right) \Delta$ as in Definition 4.1 but on $A$ and $\mathrm{d} a=\sum_{i}\left(\partial^{i} a\right) e_{i}$. The global symbols $\sigma^{i}: A \rightarrow k$ defined by $\sigma^{i}=\left\langle f^{i}, \varpi \pi_{\varepsilon}()\right\rangle$ can be recovered from $\partial^{i}$ as $\sigma^{i}(a)=\varepsilon \partial^{i} a$ and can typically be realised as evaluation against some element $x^{i}$ of a dually paired 'enveloping algebra' Hopf algebra and in this context we will write $\sigma^{i}=\sigma_{x^{i}}=\left\langle x_{i},\right\rangle$. Similarly for each $i, j$ let $C_{i}^{j}(a)=a_{(1)}\left\langle f^{j}, e_{i} \triangleleft a_{(2)}\right\rangle$, for $a \in A$, be left-invariant operators encoding the bimodule commutation relations. They have no classical analogue (they would be the identity). Their symbols $\sigma_{i}{ }^{m}: A \rightarrow k$ defined by $\sigma_{i}^{j}(a)=\left\langle f^{j}, e_{i} \triangleleft a\right\rangle=\varepsilon C_{i}{ }^{j}(a)$ can typically be given by evaluation against elements $y_{i}{ }^{j}$ of a dually paired Hopf algebra and this in this context we will write $\sigma_{i}{ }^{j}=\sigma_{y_{i}}{ }^{j}=\left\langle y_{i}{ }^{j},\right\rangle$. It is these global symbols which we extract from the algebraic structure of the calculus and need in what follows.

Now let $(\mathbb{G}, \Delta)$ be a compact quantum group with dense $*$-Hopf subalgebra $A=$ $\mathbb{C}[\mathbb{G}]$. We suppose that we have a left covariant calculus on $\mathbb{C}[\mathbb{G}]$ and remember from it the key information $\Lambda^{1}$ and the operators $\partial^{i}, C_{i}{ }^{j}$ defining the exterior derivative and bimodule relations respectively.

Proposition 7.4. Let $(\mathbb{G}, \Delta)$ be a compact quantum group and let $\left(\Omega^{1}, d\right)$ be a $n$ dimensional left-covariant differential calculus over the dense Hopf $*$-algebra $\mathbb{C}[\mathbb{G}]$ of $\mathbb{G}$. Then $\partial^{i}, C_{i}{ }^{j}$ extend to left-coinvariant operators $C_{\mathcal{D}}^{\infty}(\mathbb{G}) \rightarrow C_{\mathcal{D}}^{\infty}(\mathbb{G})$ and define a differential calculus on the algebra $C_{\mathcal{D}}^{\infty}(\mathbb{G})$ if and only if there exists $\gamma>0$ such that

$$
\max _{i, j=1, \ldots, n}\left\{\left\|\sigma_{\partial^{i}}(\pi)\right\|_{\mathrm{HS}}^{2},\left\|\sigma_{C_{i}^{j}}(\pi)\right\|_{\mathrm{HS}}^{2}\right\} \leq\left|\lambda_{\pi}\right|^{\gamma} .
$$

The extension is given by $\Omega^{1}\left(C_{\mathcal{D}}^{\infty}(\mathbb{G})\right)=C_{\mathcal{D}}^{\infty}(\mathbb{G}) \otimes \Lambda^{1}=C_{\mathcal{D}}^{\infty}(\mathbb{G}) \otimes \mathbb{C}[\mathbb{G}] \Omega^{1}$ with $\mathrm{d} a=\sum_{i}\left(\partial^{i} a\right) e_{i}$ and $e_{i} \cdot a=\sum_{j} C_{i}{ }^{j}(a) e_{j}$. 
Proof of Proposition 7.4. From the linearity of the exterior derivative $\mathrm{d}$ in the Fourier expansion, we have

$$
\mathrm{d} a=\sum_{\pi \in \widehat{\mathbb{G}}} d_{\pi} \operatorname{Tr}\left(\left(Q^{\pi}\right)^{-1}(\mathrm{~d} \pi) \widehat{a}(\pi)\right),
$$

where from the results above including Theorem 4.3 in the algebraic form on $\mathbb{C}[\mathbb{G}]$,

$$
\begin{aligned}
\mathrm{d}\left(\pi_{i j}\right) & =\sum_{k=1}^{n} \partial^{k}\left(\pi_{i j}\right) e_{k}, \\
\partial^{k} \pi_{i j} & =\sum_{m} \pi_{i m} \sigma_{\partial^{k}}(\pi)_{m j}, \\
\sigma_{\partial^{k}}(\pi)_{m j} & =\sigma^{k}\left(\pi_{m j}\right)=\pi_{m j}\left(x^{k}\right),
\end{aligned}
$$

and where the last step is the matrix of the representation of a dually paired Hopf algebra defined by $\pi$ when such $x^{k}$ exist.

Therefore, it is sufficient to check that $\partial^{k}: C_{\mathcal{D}}^{\infty} \rightarrow C_{\mathcal{D}}^{\infty}$ are continuous linear maps with respect to the topology defined by seminorms (6.2). By [Tre67, Proposition 7.7, p.64], the linear maps $\partial^{k}$ act continuously in $C_{\mathcal{D}}^{\infty}(\mathbb{G})$ if and only if for every $\alpha>0$ there is $\beta>0$ such that

$$
\sum_{\pi \in \widehat{\mathbb{G}}} d_{\pi}\left|\lambda_{\pi}\right|^{2 \alpha}\left\|\widehat{\partial^{k}(a)}\right\|_{\mathrm{HS}}^{2} \leq \sum_{\pi \in \widehat{\mathbb{G}}} d_{\pi}\left|\lambda_{\pi}\right|^{2 \beta}\|\widehat{a}\|_{\mathrm{HS}}^{2}
$$

for every $a \in C_{\mathcal{D}}^{\infty}(\mathbb{G})$.

It is clear that condition (7.1) implies (7.3). Hence, we concentrate on necessity. Taking $a=\pi_{i j} \in \widehat{\mathbb{G}}$ in (7.3), we get

$$
\lambda_{\pi}^{2 \alpha}\left\|\widehat{\partial^{k}\left(\pi_{i j}\right)}(\pi)\right\|_{\mathrm{HS}}^{2} \leq \lambda_{\pi}^{2 \beta}\left\|\widehat{\pi_{i j}}(\pi)\right\|_{\mathrm{HS}}^{2}=\lambda_{\pi}^{2 \beta} .
$$

From (7.4) dividing by $\left|\lambda_{\pi}\right|^{2 \alpha}$, we get

$$
\left\|\widehat{\partial^{k}\left(\pi_{i j}\right)}(\pi)\right\|_{\mathrm{HS}}^{2} \leq\left|\lambda_{\pi}\right|^{2(\beta-\alpha)} .
$$

From the algebraic version of Theorem 4.3 we have

$$
\widehat{\partial^{k}\left(\pi_{i j}\right)}(\pi)_{m n}=\sum_{s=1}^{n_{\pi}} \sigma_{\partial^{k}}(\pi)_{m s} \widehat{\pi_{i j}}(\pi)_{s n}=\sigma_{\partial^{k}}(\pi)_{m j} \frac{q_{i}^{\pi}}{d_{\pi}} \delta_{n i},
$$

where we used

$$
\widehat{\pi_{i j}}(\pi)_{s n}=\frac{q_{i}^{\pi}}{d_{\pi}} \delta_{s j} \delta_{n i} .
$$

The latter follows from the Peter-Weyl orthogonality relations (2.3). Hence, we get

$$
\left\|\widehat{\partial^{k}\left(\pi_{i j}\right)}\right\|_{\mathrm{H}, \mathrm{S}}^{2}=\sum_{m, n=1}^{n_{\pi}} \frac{1}{q_{m}^{\pi}}\left|\sigma_{\partial^{k}}(\pi)_{m j} \frac{q_{i}^{\pi}}{d_{\pi}} \delta_{n i}\right|^{2} .
$$

Thus, estimate (7.4) reduces to

$$
\left\|\sigma_{\partial^{k}}(\pi)\right\|_{\mathrm{HS}} \leq \lambda_{\pi}^{\beta-\gamma}, \quad \pi \in \widehat{\mathbb{G}}
$$


with $\gamma=\beta-\alpha$.

We similarly need to extend the bimodule relations from $\mathbb{C}[\mathbb{G}]$ to $C_{\mathcal{D}}^{\infty}(\mathbb{G})$ and we do this in just the same way by

$$
e_{i} \cdot a=\sum_{\pi \in \widehat{\mathbb{G}}} d_{\pi} \operatorname{Tr}\left(\left(Q^{\pi}\right)^{-1}\left(e_{i} \cdot \pi\right) \widehat{a}(\pi)\right),
$$

where from the above and the algebraic form of Theorem 4.3 we have

$$
\begin{aligned}
e_{i} . \pi_{k l} & =\sum_{j} C_{i}^{j}\left(\pi_{k l}\right) e_{j}, \\
C_{i}{ }^{j}\left(\pi_{k l}\right) & =\sum_{m} \pi_{k m} \sigma_{C_{i}}\left(\pi_{m l}\right), \\
\sigma_{C_{i} j}\left(\pi_{m l}\right) & ={\sigma_{i}}^{j}\left(\pi_{m l}\right)=\pi_{m l}\left(y_{i}^{j}\right),
\end{aligned}
$$

and where the last step is the matrix of the representation of a dually paired Hopf algebra defined by $\pi$ when such $y_{i}{ }^{j}$ exist. As before we need these linear maps $C_{i}{ }^{j}: \mathbb{C}[\mathbb{G}] \rightarrow$ $\mathbb{C}[\mathbb{G}]$ to extend to $\mathbb{C}_{\mathcal{D}}^{\infty}$ which is another Hilbert-Schmidt condition on the symbols of the same type as for the $\partial^{i}$.

Conversely, given a differential calculus $\left(\Omega^{1}, \mathrm{~d}\right)$ over the Hopf-subalgebra $\mathbb{C}[\mathbb{G}]$ of $\mathbb{G}$, we shall view (7.1) as a restriction on the $\left\{\lambda_{\pi}\right\}$ i.e. on a 'Dirac operator' $\mathcal{D}: L^{2}(\mathbb{G}) \rightarrow$ $L^{2}(\mathbb{G})$ for it to agree with the differential calculus $\left(\Omega^{1}, \mathrm{~d}\right)$ over $\mathbb{C}[\mathbb{G}]$.

Definition 7.5. Let $\mathcal{D}: L^{2}(\mathbb{G}) \rightarrow L^{2}(\mathbb{G})$ be unbounded operator given by $\mathcal{D} \pi_{m n}=$ $\lambda_{\pi} \pi_{m n}$ for some collection $\left\{\lambda_{\pi}\right\}_{\pi \in \widehat{\mathbb{G}}}$. We shall say that $\mathcal{D}$ is admissible with respect to a differential calculus $\left(\Omega^{1}, \mathrm{~d}\right)$ on $\mathbb{C}[\mathbb{G}]$ if and only if the condition (7.1) on $\left\{\lambda_{\pi}\right\}_{\pi \in \widehat{\mathbb{G}}}$ holds for some $\gamma>0$.

Whether or not an admissible $\mathcal{D}$ exists depends on the quantum group and the calculus. We look at $\mathrm{SU}_{2}^{\mathrm{q}}$ with its two main calculi of interest, the $3 \mathrm{D}$ and the $4 \mathrm{D}$ (both of these calculi are from [Wor89] but the 4D one generalises to other $q$-deformation quantum groups). As a first step, we will recall the computation of the global symbols for the vector fields on the classical $\mathrm{SU}_{2}$. We first briefly recall representation theory of $\mathrm{SU}_{2}^{\mathrm{q}}$ $\left[\mathrm{MMN}^{+} 91\right]$. The unitary dual $\widehat{\mathrm{SU}_{2}^{\mathrm{q}}}$ is parametrised by the half-integers $\frac{1}{2} \mathbb{N}_{0}$, i.e.

$$
\widehat{\mathrm{SU}_{2}^{\mathrm{q}}}=\left\{t^{l}\right\}_{l \in \frac{1}{2} \mathbb{N}_{0}} \text {. }
$$

The Peter-Weyl theorem obtained in $\left[\mathrm{MMN}^{+} 91\right.$, Theorem 3.7] allows us to describe the Fourier transform explicitly. For each $a \in \mathbb{C}\left[\mathrm{SU}_{2}^{\mathrm{q}}\right]$, we define its matrix-valued Fourier coefficient at $t^{l}$ by

$$
\widehat{a}(l)=h\left(a S t^{l}\right), \text { i.e. } \widehat{a}(l)_{m n}=h\left(a S t_{m n}^{l}\right),
$$

where $t^{l}=\left(t_{m n}^{l}\right)_{m, n \in I_{l}}$ and $I_{l}=\{-l,-l+1, \ldots,+l-1,+l\}, l \in \frac{1}{2} \mathbb{N}_{0}$. It is convenient for the inverse Fourier transform to introduce $q$-traces

$$
\tau_{l}(\sigma(l)):=\sum_{i \in I_{l}} q^{2 i} \sigma(l)_{i i} .
$$


Moreover, the $q$-trace $\tau_{l}$ naturally leads to the $q$-hermitian inner form

$$
\left(\sigma_{1}(l), \sigma_{2}(l)\right):=\tau_{l}\left(\sigma_{1}(l) \sigma_{2}(l)^{*}\right) .
$$

The Fourier inversion formula takes $\left[\mathrm{MMN}^{+} 91\right.$, Theorem 3.10] the form

$$
h\left(a b^{*}\right)=\sum_{l \in \frac{1}{2} \mathbb{N}_{0}}[2 l+1]_{q} \sum_{i, k \in I_{l}} q^{2 i} \widehat{a}(l)_{i k} \widehat{\widehat{b}(l)_{i k}}
$$

Let us denote by $C^{\infty}\left(\mathrm{SU}_{2}\right)$ the space of infinitely differentiable functions on $\mathrm{SU}_{2}$. Let $X_{+}=\left(\begin{array}{ll}0 & 0 \\ 1 & 0\end{array}\right), X_{-}=\left(\begin{array}{ll}0 & 1 \\ 0 & 0\end{array}\right), H=\frac{1}{2}\left(\begin{array}{cc}-1 & 0 \\ 0 & +1\end{array}\right)$ be a basis in the Lie algebra $\mathfrak{s u}_{2}$ of $S U(2)$ with $\left[X_{+}, X_{-}\right]=H$ and associated first-order partial differential operators

$$
\partial_{+}, \partial_{-}, \partial_{+}: C^{\infty}(S U(2)) \rightarrow C^{\infty}(S U(2))
$$

(called creation, annihilation and neutral operators, respectively, in [RT13]). Then classically, in our current conventions, one has the following.

Proposition 7.6 ([RT13, Theorem 5.7, p.2461]).

$$
\begin{aligned}
& \partial_{+} t_{m n}^{l}=\sqrt{(l-n)(l+n+1)} t_{m n+1}^{l}, \\
& \partial_{-} t_{m n}^{l}=\sqrt{(l+n)(l-n+1)} t_{m n-1}^{l}, \\
& \partial_{0} t_{m n}^{l}=n t_{m n}^{l} .
\end{aligned}
$$

From this the classical global symbols $\sigma_{\partial_{ \pm}}, \sigma_{\partial_{0}}$ can be read off as the matrix entries of $X_{ \pm}, H$ in the representation $t^{l}$. The corepresentation theory of $\mathrm{SU}_{2}^{\mathrm{q}}$ is strikingly similar to its classical counterpart giving similar results. We compute the symbols for the action of $X_{-}, X_{+}, q^{\frac{H}{2}}$ as elements of the quantum enveloping algebra $U_{q}\left(s u_{2}\right)$ acting by the regular representation on $\mathbb{C}_{q}\left[S U_{2}\right]$ and in the conventions of [Maj95].

Lemma 7.7. We have

$$
\begin{aligned}
\sigma_{X_{+}}\left(t^{l}\right)_{m n} & =\sqrt{[l-n]_{q}[l+n+1]_{q}} \delta_{m n+1}, \\
\sigma_{X_{-}}\left(t^{l}\right)_{m n} & =\sqrt{[l+n]_{q}[l-n+1]_{q}} \delta_{m n-1}, \\
\sigma_{q^{\frac{H}{2}}}\left(t^{l}\right)_{m n} & =q^{n} \delta_{m n}
\end{aligned}
$$

where $[n]_{q}=\frac{q^{n}-q^{-n}}{q-q^{-1}}$.

Proof of Lemma 7.7. Let $q$ be real and for each $l \in \frac{1}{2} \mathbb{N}_{0}$, the quantum group $\mathrm{U}_{q}(s u 2)$ has $2 l+1$-dimensional unitary representation space $V_{l}=\{|l m\rangle\}_{m=-l}^{+l}$ detailed in the relevant conventions in [Maj95, Proposition 3.2.6, p.92] so that, for example, $X_{+}|l, m\rangle=$ $\sqrt{[l-n]_{q}[l+n+1]_{q}}|l, m+1\rangle$. By definition, the $t_{m n}^{l}$ are the matrix elements of this representation, immediately giving $\sigma_{X}\left(t^{l}\right)_{m n}=\left\langle X, t_{m n}^{l}\right\rangle=t^{l}(X)_{m n}$ for the symbol of any left-invariant operator $\tilde{X}(a)=a_{(1)}\left\langle X, a_{(2)}\right\rangle$. Thus we can read off the $\sigma_{X_{ \pm}}, \sigma_{q^{\frac{H}{2}}}$ as stated. 
For the convenience of the reader we recall that the $Q$-matrix for $\mathbb{G}=\mathrm{SU}_{2}^{\mathrm{q}}$ is given $\left[\mathrm{MMN}^{+} 91\right]$ by

$$
Q^{l}=\operatorname{diag}\left(q^{-2 i}\right)_{i=-l}^{l}, \quad l \in \frac{1}{2} \mathbb{N}_{0} .
$$

As a warm-up we look at the admissibility condition (7.1) of Proposition 7.4.

Lemma 7.8. Let $b_{q}=\max \left(q, \frac{1}{q}\right)$. Then

$$
[n]_{q} \cong b_{q}^{n} .
$$

We write $x \cong y$ if there are constants $c_{1}, c_{2} \neq 0$ such that

$$
c_{1} x \leq y \leq c_{2} x
$$

Lemma 7.9. Let $\mathbb{G}=\mathrm{SU}_{2}^{\mathrm{q}}$. Then we have

$$
\begin{aligned}
\left\|\sigma_{X_{+}}\left(t^{l}\right)\right\|_{\mathrm{HS}} & \lesssim[2 l+1]_{q}, \\
\left\|\sigma_{X_{-}}\left(t^{l}\right)\right\|_{\mathrm{HS}} & \lesssim[2 l+1]_{q}, \\
\left\|\sigma_{q^{\frac{H}{2}}}\left(t^{l}\right)\right\|_{\mathrm{HS}} & \lesssim[2 l+1]_{q} .
\end{aligned}
$$

Proof of Lemma 7.9. By (2.8)

$$
\begin{aligned}
\left\|\sigma_{X_{+}}\left(t^{l}\right)\right\|_{\mathrm{HS}}^{2} & :=\sum_{m=-l}^{+l} q^{2 m} \sum_{n=-l}^{+l}\left|\sigma_{X_{+}}\left(t^{l}\right)_{m n}\right|^{2}=\sum_{m=-l}^{+l} q^{2 m}[l-m+1]_{q}[l+m]_{q} \\
& \cong \sum_{m=-l}^{+l} q^{2 m} b_{q}^{l-m+1} b_{q}^{l+m}=b_{q}^{2 l+1} \sum_{m=-l}^{+l} q^{2 m}=b_{q}^{4 l},
\end{aligned}
$$

where we used the fact that

$$
\sum_{m=-l}^{+l} q^{m} \cong b_{q}^{l}
$$

Similarly, we get

$$
\begin{aligned}
\left\|\sigma_{X_{-}}\left(t^{l}\right)\right\|_{\mathrm{HS}}^{2} & =\sum_{m=-l}^{+m} q^{2 m}[l+m+1]_{q}[l-m]_{q} \cong \sum_{m=-l}^{+m} q^{2 m} b_{q}^{l+m+1} b_{q}^{l-m}=b_{q}^{2 l+1} b_{q}^{2 l} \\
& \cong b_{q}^{4 l} \cong[2 l+1]_{q}^{2} .
\end{aligned}
$$

Finally, we compute

$$
\left\|\sigma_{q^{\frac{H}{2}}}\left(t^{l}\right)\right\|_{\mathrm{HS}}^{2}=\sum_{m=-l}^{+l} q^{2 m} q^{2 m} \cong b_{q}^{4 l} \cong[2 l+1]_{q}^{2} .
$$

This completes the proof. 
By the arguments as in the proof of Proposition 7.4 it follows that the associated left covariant operators to $X_{ \pm}$and $q^{\frac{H}{2}}-1$ extend to $C_{\mathcal{D}}^{\infty}\left(\mathrm{SU}_{2}^{\mathrm{q}}\right)$ where

$$
\mathcal{D} t_{i j}^{l}= \pm[2 l+1]_{q} t_{i j}^{l}
$$

since the \|\|$_{H S}^{2}$ condition similar to (7.1) holds, but for the symbols of these operators rather than for a choice of calculus.

7.1. $3 D$ calculus on $\mathrm{SU}_{2}^{\mathrm{q}}$. We are now ready for the left-covariant $3 \mathrm{D}$ calculus on $\mathbb{C}_{q}\left[S U_{2}\right]$ which we take with the defining 2-dimensional representation with $t^{\alpha}{ }_{\beta}=$ $\{a, b, c, d\}$ to give the standard matrix of generators with usual conventions where $b a=q a b$ etc. We let || denote the known $\mathbb{Z}$-grading on the algebra defined as the number of $a, c$ minus the number of $b, d$ in any monomial. The 3D calculus has generators $e_{0}, e_{ \pm}$with commutation relations

$$
e_{0} f=q^{2|f|} f e_{0}, \quad e_{ \pm} f=q^{|f|} f e_{ \pm}
$$

(which implies the action in the vector space $\Lambda^{1}$ with basis $e_{0}, e_{ \pm}$). The exterior derivative is

$$
\mathrm{d} a=a e_{0}+q b e_{+}, \quad \mathrm{d} b=a e_{-}-q^{-2} b e_{0}, \quad \mathrm{~d} c=c e_{0}+q d e_{+}, \quad \mathrm{d} d=c e_{-}-q^{-2} d e_{0}
$$

Next the combinations $\sigma^{k}=f^{k} \circ \varpi \pi_{\varepsilon}=\varepsilon \partial^{k}$ are linear functionals on $A=\mathbb{C}_{q}\left[S U_{2}\right]$ and can in fact be identified as evaluation against elements $x^{k} \in U_{q}\left(s u_{2}\right)$ in our case.

Proposition 7.10. The $3 D$ calculus $\left(\Omega_{3 D}^{1}, \mathbb{C}_{q}\left[S U_{2}\right]\right.$, d) over $\mathbb{C}_{q}\left[S U_{2}\right]$ is generated by the action of

$$
x^{+}=q^{\frac{1}{2}} X_{-} q^{\frac{H}{2}}, \quad x^{-}=q^{-\frac{1}{2}} X_{+} q^{\frac{H}{2}}, \quad x^{0}=\frac{q^{2 H}-1}{q^{2}-1}
$$

and extends to $C_{\mathcal{D}}^{\infty}\left(\mathrm{SU}_{2}^{\mathrm{q}}\right)$ where $\mathcal{D}$ defined in $(7.15)$ is admissible with $\gamma=2$. The symbols are given by

$$
\begin{aligned}
\sigma_{x^{+}}\left(t^{l}\right)_{m n} & =q^{n+\frac{1}{2}} \sqrt{[l+m+1]_{q}[l-m]_{q}} \delta_{m n+1}\left(1-\delta_{m 2 l+1}\right), \\
\sigma_{x^{-}}\left(t^{l}\right)_{m n} & =q^{n-\frac{1}{2}} \sqrt{[l-m+1]_{q}[l+m]_{q}} \delta_{m n}\left(1-\delta_{m 1}\right)_{m n}, \\
\sigma_{x^{0}}\left(t^{l}\right)_{m n} & =\frac{h\left(t_{m n}^{l}\right)-q^{4 n} \delta_{m n}}{1-q^{2}} .
\end{aligned}
$$

Proof of Proposition 7.10. The 3D calculus is constructed 'by hand' so we use the form $\sigma^{k}=\varepsilon \partial^{k}$ and the known form of the partial derivatives (obtained by computing $\mathrm{d}$ on monomials via the Leibniz rule) and find elements $x^{k} \in U_{q}\left(s u_{2}\right)$ as stated that give these. One then finds the symbols $\sigma_{x^{k}}(\pi)_{i j}=\pi\left(x^{i}\right)_{i j}$ as

$$
\begin{aligned}
\sigma_{x^{+}}\left(t^{l}\right)_{m n} & =q^{\frac{1}{2}}\left[\sigma_{X_{-}}\left(t^{l}\right) \sigma_{q^{\frac{H}{2}}}\left(t^{l}\right)\right]_{m n}=q^{\frac{1}{2}} \sum_{k=1}^{2 l+1} \sigma_{X_{-}}\left(t^{l}\right)_{m k} \sigma_{q^{\frac{H}{2}}}\left(t^{l}\right)_{k n} \\
& =\sum_{k=1}^{2 l+1} \sqrt{[l+k]_{q}[l-k+1]_{q}} \delta_{m k-1} q^{n} \delta_{k n} \\
& =q^{n+\frac{1}{2}} \sqrt{[l+m+1]_{q}[l-m]_{q}} \delta_{m n+1}\left(1-\delta_{m} 2 l+1\right),
\end{aligned}
$$


where we used the fact that $\sigma_{X}(\pi) \sigma_{Y}(\pi)=\sigma_{X Y}(\pi)$ since these are matrices for $X, Y, X Y$ in the representation $\pi$, and Lemma 7.7. Similarly, we establish

$$
\begin{aligned}
\sigma_{x^{-}}\left(t^{l}\right)_{m n} & =q^{n-\frac{1}{2}} \sqrt{[l-m+1]_{q}[l+m]_{q}} \delta_{m n}\left(1-\delta_{m 1}\right)_{m n}, \\
\sigma_{x^{0}}\left(t^{l}\right)_{m n} & =\frac{h\left(t_{m n}^{l}\right)-q^{4 n} \delta_{m n}}{1-q^{2}} .
\end{aligned}
$$

where for the haar function $h$ we estimate

$$
\left|h\left(t_{m n}^{l}\right)\right| \leq\left\|t_{m n}^{l}\right\|_{\mathrm{op}} h(1) \leq h(1) .
$$

It is then straightforward to check that the condition (7.1) is satisfied for the symbols $\sigma_{x^{ \pm}}\left(t^{l}\right), \sigma_{x^{0}}\left(t^{l}\right)$. Hence, the application of Proposition 7.4 shows that the vector fields $x_{ \pm}, x_{0}$ are continuous.

Now, we check condition (7.1) allowing us to extend $x^{+}, x^{-}, x^{0}$ continuously. We have

$$
\begin{aligned}
\left\|\sigma_{x^{+}}\left(t^{l}\right)\right\|_{\mathrm{HS}}^{2} & =\sum_{m, n=-l}^{+l} q^{2 n+1}[l+m+1]_{q}[l-m]_{q} \cong \sum_{m, n=-l}^{+l} q^{2 n+1} b_{q}^{l+m+1} b_{q}^{l-m} \\
& =b_{q}^{2 l+1} \sum_{m, n=-l}^{+l} q^{2 n+1} \cong b_{q}^{2 l+1} b^{2 l} \cong b_{q}^{4 l} .
\end{aligned}
$$

Similarly

$$
\left\|\sigma_{x^{-}}\left(t^{l}\right)\right\|_{\mathrm{HS}}^{2}=\sum_{m, n=-l}^{+l} q^{2 n-1}[l-m+1]_{q}[l+m]_{q} \cong b_{q}^{4 l} .
$$

We similarly have commutation relations given for $i, j= \pm$ by

$$
\sigma_{i}{ }^{j}\left(t^{l}\right)_{m n}=\delta_{i j} t^{l}\left(y^{i}\right)_{m n}=\delta_{i j} \delta_{m n}\left\{\begin{array}{ll}
q^{-2 m} & i= \pm \\
q^{-4 m} & i=0
\end{array} ; \quad y^{ \pm}=q^{-H}, y^{0}=q^{-2 H}\right.
$$

if we number the indices by $-l, \ldots, l$ for the $2 l+1$ dimensional representation $t^{l}$. This gives commutation relations $e_{i} t_{j k}^{l}=t_{j m}^{l} \sigma_{i}{ }^{p}\left(t^{l}\right)_{m k} e_{p}=t_{j m}^{l} e_{i} \delta_{m k} q^{-2 k}=t_{j k}^{l} q^{-2 k} e_{i}$ for $i= \pm\left(\right.$ and $q^{2}$ in place of $q$ if $i=0$ ) which corresponds to a $\mathbb{Z}$-grading of $\mathbb{C}_{q}\left[S U_{2}\right]$ where $t_{j k}^{l}$ has grade $-2 k$. For the spin $1 / 2$ representation it means $a, c$ in the standard matrix generators $t_{m n}$ of the quantum group have grade 1 and $b, d$ have grade -1 as expected. We compute $\left\|\sigma_{i}{ }^{j}\left(t^{l}\right)\right\|_{\mathrm{HS}}^{2}$ similarly as in the proof of Lemma 7.9. By (7.21)

$$
\begin{aligned}
\left\|\sigma_{i}{ }^{j}\left(t^{l}\right)\right\|_{\mathrm{HS}}^{2} & :=\sum_{n=-l}^{+l} q^{2 n} \sum_{m=-l}^{+l}\left|q^{-2 n} \delta_{m n} \delta_{i j}\right|^{2} \\
& =\delta_{i j} \sum_{n=-l}^{+l}=(2 l+1) \leq b_{q}^{2 l+1} \cong[2 l+1]_{q} .
\end{aligned}
$$


7.2. $4 \mathrm{D}$ calculus on $\mathrm{SU}_{2}^{\mathrm{q}}$. As before we denote the standard $2 \times 2$ matrix of generators of $\mathbb{C}_{q}\left[S U_{2}\right]$ by $a, b, c, d$. This time (from the general construction given later or from [Wor89]) there is a basis $e_{a}, e_{b}, e_{c}, e_{d}$ corresponding to the generators, with relations and exterior derivative

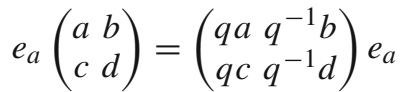

$$
\begin{aligned}
& {\left[e_{b},\left(\begin{array}{ll}
a & b \\
c & d
\end{array}\right)\right]=q \lambda\left(\begin{array}{ll}
0 & a \\
0 & c
\end{array}\right) e_{a}, \quad\left[e_{c},\left(\begin{array}{ll}
a & b \\
c & d
\end{array}\right)\right]=q \lambda\left(\begin{array}{ll}
b & 0 \\
d & 0
\end{array}\right) e_{a}} \\
& {\left[e_{d},\left(\begin{array}{l}
a \\
c
\end{array}\right)\right]_{q^{-1}}=\lambda\left(\begin{array}{l}
b \\
d
\end{array}\right) e_{b}, \quad\left[e_{d},\left(\begin{array}{l}
b \\
d
\end{array}\right)\right]_{q}=\lambda\left(\begin{array}{l}
a \\
c
\end{array}\right) e_{c}+q \lambda^{2}\left(\begin{array}{l}
b \\
d
\end{array}\right) e_{a},} \\
& \mathrm{~d}\left(\begin{array}{l}
a \\
c
\end{array}\right)=\left(\begin{array}{l}
a \\
c
\end{array}\right)\left((q-1) e_{a}+\left(q^{-1}-1\right) e_{d}\right)+\lambda\left(\begin{array}{l}
b \\
d
\end{array}\right) e_{b} \\
& \mathrm{~d}\left(\begin{array}{l}
b \\
d
\end{array}\right)=\left(\begin{array}{l}
b \\
d
\end{array}\right)\left(\left(q^{-1}-1+q \lambda^{2}\right) e_{a}+(q-1) e_{d}\right)+\lambda\left(\begin{array}{l}
a \\
c
\end{array}\right) e_{c} .
\end{aligned}
$$

Here $[x, y]_{q} \equiv x y-q y x$ and $\lambda=1-q^{-2}$.

Then the generators of the calculus are elements $x^{k} \in U_{q}\left(s u_{2}\right)$ where $k=a, b, c, d$ which we organise as a $2 \times 2$ matrix of elements $\left(x^{\alpha \beta}\right)$ where

$$
\left(x^{\alpha \beta}\right)=\left(\begin{array}{cc}
q^{H}+q \lambda^{2} X_{-} X_{+}-1 & q^{\frac{1}{2}} \lambda X_{-} q^{-\frac{H}{2}} \\
q^{\frac{1}{2}} \lambda q^{-\frac{H}{2}} X_{+} & q^{-H}-1
\end{array}\right)
$$

These combinations $x^{\alpha \beta}$ are known to span the right handed braided-Lie algebra $L \subset$ $U_{q}\left(s u_{2}\right)$ and generate the quantum group [Maj15].

Proposition 7.11. The $4 D$ calculus $\left(\Omega_{4 D}^{1}, \mathbb{C}_{q}\left[S U_{2}\right], \mathrm{d}\right)$ extends to $C_{\mathcal{D}}^{\infty}\left(\mathrm{SU}_{2}^{\mathrm{q}}\right)$ for the same $\mathcal{D}$ in (7.15), which is again admissible with $\gamma=2$.

Proof of Proposition 7.11. We compute the symbol $\sigma_{x^{\alpha \beta}}(\pi)$ of $x^{\alpha \beta}$ composing the results in Lemma 7.7 to find

$$
\begin{aligned}
& \sigma_{\left(x^{\alpha \beta}\right)}\left(t_{m n}^{l}\right) \\
& \quad=\left(\begin{array}{cc}
\left(q^{2 l}+q^{-2 l-2}-q^{-2 n-2}-1\right) \delta_{m n} & q^{-n+\frac{1}{2}} \lambda \sqrt{[l+n]_{q}[l-n+1]_{q}} \delta_{m n-1} \\
q^{-n-\frac{1}{2}} \lambda \sqrt{[l-n]_{q}[l+n+1]_{q}} \delta_{m n+1} & \left(q^{-2 n}-1\right) \delta_{m n}
\end{array}\right)
\end{aligned}
$$

It is sufficient to check that $x^{\alpha \beta}$ acts continuously in each $L^{2}(\mathbb{G})$. Let us denote

$$
\sigma_{\left(x^{\alpha \beta}\right)}\left(t^{l}\right)=\left(\begin{array}{ll}
\sigma^{a}\left(t^{l}\right) & \sigma^{b}\left(t^{l}\right) \\
\sigma^{c}\left(t^{l}\right) & \sigma^{d}\left(t^{l}\right)
\end{array}\right) .
$$

By (2.8)

$$
\left\|\sigma\left(t^{l}\right)\right\|_{\mathrm{HS}}^{2}=\sum_{m=-l}^{+l} q^{2 m} \sum_{n=-l}^{+l}\left|\sigma\left(t^{l}\right)_{m n}\right|^{2} .
$$


Composing (7.21) and (7.20), we get

$$
\begin{aligned}
\left\|\sigma^{a}\left(t^{l}\right)\right\|_{\mathrm{HS}}^{2}= & \sum_{m=-l}^{+l} q^{2 m} \sum_{n=-l}^{+l}\left|\sigma^{a}\left(t^{l}\right)_{m n}\right|^{2} \\
= & \sum_{m=-l}^{+l} q^{2 m}\left(q^{2 l}+q^{-2 l-2}-q^{-2 m-2}-1\right)^{2} \\
= & \sum_{m=-l}^{+l}\left(q^{2} l+q^{-2 l-2}\right)^{2} \\
& -\sum_{m=-l}^{+l} 2\left(q^{2 l}+q^{-2 l-2}\right)\left(q^{2 m-2}-1\right)+\left(q^{-2 m-2}-1\right)^{2} \\
= & \sum_{m=-l}^{+l} q^{4 l}+2 q^{2 l-2 l-2}+q^{-4 l-4} \\
& -\sum_{m=-l}^{+l} 2\left(q^{2 l-2 m-2}-q^{2 l}+q^{-2 l-2 m-4}-q^{-2 l-2}\right) \\
& +\left(-2 q^{2 l-2}-2 q^{-2 l-4}-2 q^{-2}\right) \sum_{m=-l}^{+l} q^{-2 m}+q^{-4} \sum_{m=-l}^{+l} q^{-4 m} \\
= & \left(q^{4 l}+2 q^{-4}+q^{4 l-4}+2 q^{2 l}+2 q^{-2 l-2}+1\right)(2 l+1) \\
& +
\end{aligned}
$$

The expression $-2 q^{2 l-2}-2 q^{-2 l-4}-2 q^{-2}$ is always negative. Therefore, we get

$$
\begin{aligned}
\left\|\sigma^{a}\left(t^{l}\right)\right\|_{\mathrm{HS}}^{2} \leq & \left(q^{4 l}+2 q^{-2}+q^{-4 l-4}+2 q^{2 l}+2 q^{-2 l-2}+1\right)(2 l+1) \\
& +q^{-4} \sum_{m=-l}^{+l} q^{-4 m} .
\end{aligned}
$$

It is straightforward to check that

$$
q^{4 l}+2 q^{-2}+q^{-4 l-4}+2 q^{2 l}+2 q^{-2 l-2}+1 \cong b_{q}^{4 l}
$$

and

$$
\sum_{m=-l}^{+l} q^{-4 m}=\sum_{m=-l}^{+l} q^{4 m} \cong b_{q}^{4 l} .
$$

Using (7.23) and (7.24), we get from (7.22)

$$
\left\|\sigma^{a}\left(t^{l}\right)\right\|_{\mathrm{HS}}^{2} \lesssim b_{q}^{4 l}(2 l+1) \lesssim b_{q}^{5 l} \cong\left(b_{q}^{2 l+1}\right)^{\frac{5}{2}} \cong[2 l+1]_{q}^{\frac{5}{2}},
$$


where in the first inequality we used the fact

$$
(2 l+1) \lesssim b_{q}^{l} .
$$

In the second inequality we used Lemma 7.8 with $n=2 l+1$. Now, we compute $\left\|\sigma^{b}\left(t^{l}\right)\right\|_{\mathrm{HS}}^{2}$

$$
\begin{aligned}
\left\|\sigma^{b}\left(t^{l}\right)\right\|_{\mathrm{HS}}^{2} & =\sum_{m=-l}^{+l} q^{2 m} \sum_{n=-l}^{+l}\left|q^{-n+\frac{1}{2}} \lambda \sqrt{[l+n]_{q}[l-n+1]_{q}} \delta_{m, n-1}\right|^{2} \\
& =\sum_{m=-l}^{+l} q^{2 m} q^{-2 m} q^{-1} \lambda^{2}[l+m+1]_{q}[l-m]_{q} \cong \sum_{m=-l}^{+l}[l+m+1]_{q}[l-m]_{q} \\
& \cong \sum_{m=-l}^{+l} b_{q}^{l+m+1} b_{q}^{l-m}=\sum_{m=-l}^{+l} b_{q}^{2 l}=(2 l+1) b_{q}^{2 l} \lesssim b_{q}^{4 l} \cong[2 l+1]_{q}^{2} .
\end{aligned}
$$

We can argue analogously for $\left\|\sigma^{c}\left(t^{l}\right)\right\|_{\mathrm{HS}}$ to get

$$
\left\|\sigma^{b}\left(t^{l}\right)\right\|_{\mathrm{HS}}^{2} \lesssim[2 l+1]_{q}^{2} .
$$

Finally, one checks by direct calculation that

$$
\begin{aligned}
\left\|\sigma^{d}\left(t^{l}\right)\right\|_{\mathrm{HS}}^{2} & =\sum_{m=-l}^{+l} q^{2 m}\left|\left(q^{-2 n}-1\right) \delta_{m n}\right|^{2}=\sum_{m=-l}^{+l} q^{2 m}\left(q^{-4 m}-2 q^{-2 m}+1\right) \\
& =\sum_{m=-l}^{+l} q^{-2 m}-2(2 l+1)+\sum_{m=-l}^{+l} q^{2 m} \leq 2 \sum_{m=-l}^{+l} q^{2 m} \cong b_{q}^{2 l} \cong[2 l+1]_{q} .
\end{aligned}
$$

Now, we check that the matrices $\sigma_{\alpha \beta} \gamma \delta$ encoding the bimodule commutation relations in the $4 D$ calculus satisfy condition (7.1) with some exponent $\gamma$. The bimodule relations are best handled as part of a general construction discussed later and from (7.30) and (7.31) there, we see the seven values

$$
\sigma_{j 1}^{i 2}\left(t^{l}\right)_{m n}=\sigma_{\left(S l^{-i}\right) l^{+1}{ }_{2}}\left(t^{l}\right)_{m n}=0, \quad \sigma_{1 i}^{2 j}\left(t^{l}\right)_{m n}=\sigma_{\left(S l^{-2}\right)_{l} l^{+i}{ }_{j}}\left(t^{l}\right)_{m n}=0
$$

since $l_{2}^{+1}=l^{-2}{ }_{1}=0$. The non-zero matrices $\sigma_{\alpha \beta}{ }^{\gamma \delta}$ are obtained by reading (7.31) and plugging it into (7.30), noting that $S X_{-}=-q^{-1} X_{-} S q^{\frac{H}{2}}=q^{-\frac{H}{2}}$ for the action of the antipode. We then compute the symbols by composing the symbols for the composition of invariant operators, to obtain

$$
\begin{aligned}
\sigma_{11}^{11}\left(t^{l}\right)_{m n} & =\sigma_{\left(S l^{-1} 1\right) l^{+1} 1}\left(t^{l}\right)_{m n}=\sigma_{q^{\frac{H}{2}} q^{\frac{H}{2}}}\left(t^{l}\right)_{m n}=q^{2 n} \delta_{m n}, \\
\sigma_{12}{ }^{11}\left(t^{l}\right)_{m n} & =\sigma_{\left(S l^{-1} 1\right) l^{+2}{ }_{1}}\left(t^{l}\right)_{m n}=\sigma_{q^{\frac{H}{2}} q^{-\frac{1}{2}}\left(q-q^{-1}\right) X_{+}}\left(t^{l}\right)_{m n} \\
& =q^{-\frac{1}{2}}\left(q-q^{-1}\right) q^{m} \sqrt{[l-m]_{q}[l+m+1]_{q}} \delta_{m, n+1}, \\
\sigma_{12}{ }^{12}\left(t^{l}\right)_{m n} & =\sigma_{\left(S l^{-1}\right)_{l} l_{2}{ }_{2}\left(t^{l}\right)_{m n}}=\sigma_{q^{\frac{H}{2}} \cdot q^{-\frac{H}{2}}}\left(t^{l}\right)_{m n}=\delta_{m n},
\end{aligned}
$$




$$
\begin{aligned}
\sigma_{21}{ }^{11}\left(t^{l}\right)_{m n} & =\sigma_{\left(S l^{-1}{ }_{2}\right) l^{+1}{ }_{1}}\left(t^{l}\right)_{m n}=\sigma_{q^{-\frac{1}{2}}\left(q-q^{-1}\right) X_{-} \cdot q^{\frac{H}{2}}}\left(t^{l}\right)_{m n} \\
& =q^{-\frac{1}{2}}\left(q-q^{-1}\right) \sqrt{[l+n]_{q}[l-n+1]_{q}} q^{n} \delta_{m, n-1}, \\
\sigma_{22}{ }^{11}\left(t^{l}\right)_{m n} & =\sigma_{\left(S l^{-1}{ }_{2}\right) l^{+2}{ }_{1}}\left(t^{l}\right)_{m n}=\sigma_{q^{-\frac{1}{2}}\left(q-q^{-1}\right) X_{-} \cdot q^{-\frac{1}{2}}}\left(q-q^{-1}\right)_{X_{+}}\left(t^{l}\right)_{m n} \\
& =q^{-1}\left(q-q^{-1}\right)^{2} \sqrt{[l+m]_{q}[l-m+1]_{q}} \sqrt{[l-n]_{q}[l+n+1]_{q}} \delta_{m n},
\end{aligned}
$$

$$
\begin{aligned}
& \sigma_{22}^{12}\left(t^{l}\right)_{m n}=\sigma_{\left(S l^{-1}{ }_{2}\right) l^{+2}{ }_{2}}\left(t^{l}\right)_{m n}=\sigma_{q^{-\frac{1}{2}}\left(q-q^{-1}\right) X_{-} \cdot q^{-\frac{H}{2}}}\left(t^{l}\right)_{m n} \\
& =q^{-\frac{1}{2}}\left(q-q^{-1}\right) \sqrt{[l+n]_{q}[l-n+1]_{q}} q^{-n} \delta_{m, n-1} \\
& \sigma_{21}^{21}\left(t^{l}\right)_{m n}=\sigma_{\left(S l^{-2}\right)_{l}{ }^{+1}{ }_{1}}\left(t^{l}\right)_{m n}=\sigma_{q^{-\frac{H}{2}} \cdot q^{\frac{H}{2}}}\left(t^{l}\right)_{m n}=\delta_{m n}, \\
& \sigma_{22}^{21}\left(t^{l}\right)_{m n}=\sigma_{\left(S l^{-2}{ }_{2}\right) l^{+2}{ }_{1}}\left(t^{l}\right)_{m n}=\sigma_{q^{-\frac{H}{2}} \cdot q^{-\frac{1}{2}}\left(q-q^{-1}\right) X_{+}}\left(t^{l}\right)_{m n} \\
& =q^{-\frac{1}{2}}\left(q-q^{-1}\right) q^{-m} \sqrt{[l-n]_{q}[l+n+1]_{q}} \delta_{m, n+1}, \\
& \sigma_{22}^{22}\left(t^{l}\right)_{m n}=\sigma_{\left(S l^{-2}{ }_{2}\right) l^{+2}{ }_{2}}\left(t^{l}\right)_{m n}=\sigma_{q^{-\frac{H}{2}}} q^{-\frac{H}{2}}\left(t^{l}\right)_{m n}=q^{-2 n} \delta_{m n} \text {. }
\end{aligned}
$$

Now we can compute the corresponding $q$-deformed Hilbert-Schmidt norms,

$$
\begin{aligned}
& \left\|\sigma_{11} 11\left(t^{l}\right)\right\|_{\mathrm{HS}}^{2}=\sum_{-l}^{+l} q^{6 m} \cong b_{q}^{6 l} \cong[2 l+1]_{q}^{3}, \\
& \left\|\sigma_{12}{ }^{11}\left(t^{l}\right)\right\|_{\mathrm{HS}}^{2} \cong \sum_{m=-l}^{+l} q^{2 m} q^{2 m}[l-m]_{q}[l+m+1]_{q} \cong \sum_{m=-l}^{+l} q^{4 m} b_{q}^{l-m} b_{q}^{l+m+1} \\
& \cong b_{q}^{4 l} b_{q}^{2 l+1} \cong[2 l+1]_{q}^{3}, \\
& \left\|\sigma_{12}{ }^{12}\left(t^{l}\right)\right\|_{\mathrm{HS}}^{2}=\left\|\sigma_{21}{ }^{21}\left(t^{l}\right)\right\|_{\mathrm{HS}}^{2} \cong \sum_{m=-l}^{+l} q^{2 m} \cong b_{q}^{2 l} \cong[2 l+1]_{q}, \\
& \left\|\sigma_{21}{ }^{11}\left(t^{l}\right)\right\|_{\mathrm{HS}}^{2} \cong \sum_{m=-l}^{+l} q^{2 m} q^{2 m}[l+m+1]_{q}[l-m]_{q} \cong b_{q}^{2 l+1} \sum_{m=-l}^{+l} q^{4 m} \\
& \cong b_{q}^{2 l+1} b_{q}^{4 l} \cong[2 l+1]_{q}^{3}, \\
& \left\|\sigma_{22}{ }^{11}\left(t^{l}\right)\right\|_{\mathrm{HS}}^{2} \cong \sum_{m=-l}^{+l} q^{2 m}[l+m]_{q}[l-m+1]_{q}[l-m]_{q}[l+m+1]_{q} \\
& \cong \sum_{m=-l}^{+l} q^{2 m} b_{q}^{l+m} b_{q}^{l-m+1} b_{q}^{l-m} b_{q}^{l+m+1}=b_{q}^{2(2 l+1)} b_{q}^{2 l} \lesssim b_{q}^{3(2 l+1)} \cong[2 l+1]_{q}^{3}, \\
& \left\|\sigma_{22}{ }^{12}\left(t^{l}\right)\right\|_{\mathrm{HS}}^{2} \cong \sum_{m=-l}^{+l} q^{2 m} q^{-2 m}[l+m+1]_{q}[l-m]_{q} \cong \sum_{m=-l}^{+l} b_{q}^{l+m+1} b_{q}^{l-m} \\
& =(2 l+1) b_{q}^{2 l+1} \lesssim[2 l+1]_{q}^{2},
\end{aligned}
$$




$$
\begin{aligned}
\left\|\sigma_{22}{ }^{21}\left(t^{l}\right)\right\|_{\mathrm{HS}}^{2} & \cong \sum_{m=-l}^{+l} q^{2 m} q^{-2 m}[l-m+1]_{q}[l+m]_{q} \cong \sum_{m=-l}^{+l} b_{q}^{l-m+1} b_{q}^{l+m} \\
& =(2 l+1) b^{2 l+1} \lesssim[2 l+1]_{q}^{2}, \\
\left\|\sigma_{22}{ }^{22}\left(t^{l}\right)\right\|_{\mathrm{HS}}^{2} & =\sum_{m=-l}^{+l} q^{2 m} q^{-4 m}=\sum_{m=-l}^{+l} q^{-2 m}=\sum_{m=-l}^{+l} q^{2 m} \cong b_{q}^{2 l} \\
& \cong[2 l+1]_{q} .
\end{aligned}
$$

The application of Proposition 7.4 completes the proof that $\mathrm{d}$ extends.

7.3. Generalising to other coquasitriangular Hopf algebras. The bicovariant 4D calculus on $A=\mathbb{C}_{q}\left[S U_{2}\right]$ is an example of a canonical construction whenever $A$ is coquasitriangular in the dual of the sense of V.G. Drinfeld, i.e. a map $\mathcal{R}: A \otimes A \rightarrow \mathbb{C}$ obeying certain axioms. This gives a bicovariant calculus for any $L \subset A$ a subcoalgebra [Maj15]. We define

$$
\mathcal{Q}: A^{+} \otimes L \rightarrow \mathbb{C}, \quad \mathcal{Q}(a \otimes b)=\mathcal{R}\left(b_{(1)} \otimes a_{(1)}\right) \mathcal{R}\left(a_{(2)} \otimes b_{(2)}\right)
$$

which we view as $\varpi=\mathcal{Q}: A^{+} \rightarrow \Lambda^{1}=L^{*}$. If this is not surjective we take $\Lambda^{1}$ to be the image, but in examples it tends to be surjective so we suppose this as a property of the data $(A, \mathcal{R}, L)$. In addition $L$ is canonically a left crossed $A$-module [Maj15] which makes $\Lambda^{1}$ a right crossed $A$-module with $\varpi$ a morphism. Here the left action on $L$ is

$$
a \triangleright b=b_{(2)} \mathcal{R}\left(b_{(1)} \otimes a_{(1)}\right) \mathcal{R}\left(a_{(2)} \otimes b_{(3)}\right), \quad \forall a \in A, b \in L .
$$

The simplest case of interest is when $L$ is the span of the matrix elements of a corepresentation $t \in \widehat{A}, L=\operatorname{span}\left\{t^{\alpha}{ }_{\beta}\right\}$. We let $\left\{e_{\alpha \beta}\right\}$ be the dual basis of $\Lambda^{1}$, so $f^{\alpha \beta}=t^{\alpha}{ }_{\beta}$ is the dual basis element to $e_{\alpha \beta}$. We let $e_{\alpha}$ be a basis of the corepresentation $V$ so $\Delta_{R} e_{\alpha}=e_{\beta} \otimes t^{\beta}{ }_{\alpha}$. The associated left representation of any Hopf algebra $U$ dually paired to $A$ is $t(x)^{\alpha}{ }_{\beta}=\left\langle t^{\alpha}{ }_{\beta}, x\right\rangle$ for all $x \in U$ or $x . e_{\alpha}=e_{\beta}\left\langle t^{\beta}{ }_{\alpha}, x\right\rangle$. In this case if $\pi_{i j}$ are the matrix elements of a representation $\pi \in \widehat{A}$ then

$$
\sigma^{\alpha \beta}(\pi)_{i j}=f^{\alpha \beta}\left(\varpi\left(\pi_{i j}-\delta_{i j}\right)\right)=\mathcal{Q}\left(\pi_{i j} \otimes t_{\beta}^{\alpha}\right)-\delta_{i j} \delta_{\beta}^{\alpha}
$$

which we can usually write as

$$
\sigma^{\alpha \beta}(\pi)_{i j}=\pi\left(x^{\alpha \beta}\right)_{i j}, \quad \mathcal{Q}\left(a \otimes t^{\alpha}{ }_{\beta}\right)=\varepsilon(a) \delta_{\beta}^{\alpha}+\left\langle a, x^{\alpha \beta}\right\rangle, \quad \forall a \in A,
$$

for some elements $x^{\alpha \beta} \in U$ for suitable $U$. Here $x^{\alpha \beta}=\left(\left(S l^{-}\right) l^{+}\right)^{\alpha}{ }_{\beta}-\delta^{\alpha}{ }_{\beta}$ in the quantum groups literature [Maj95] for certain elements $l^{ \pm \alpha}{ }_{\beta} \in U$. These elements are evaluated in the associated matrix representation $\pi$ of $U$ and $\varepsilon\left(x^{\alpha}{ }_{\beta}\right)=0$ is implied by the above. Similarly, the adjoint of the action on $L$ gives the right action

$$
e_{\alpha \beta} \triangleleft a=e_{\gamma \delta} \mathcal{R}\left(t_{\alpha}^{\gamma} \otimes a_{(1)}\right) \mathcal{R}\left(a_{(2)} \otimes t_{\delta}^{\beta}\right)=e_{\gamma \delta}\left\langle a, S l^{-\gamma}{ }_{\alpha} l^{+\beta}\right\rangle .
$$

Hence the action of matrix elements $\pi_{i j}$ of a corepresentation is

$$
e_{\alpha \beta} \triangleleft \pi_{i j}=e_{\gamma \delta} \pi\left(\left(S l_{\alpha}^{-\gamma}\right) l_{\delta}^{+\beta}\right)_{i j},
$$


or in terms of the matrix that governs the commutation relations, this is

$$
\sigma_{\alpha \beta}^{\gamma \delta}(\pi)_{i j}=\pi\left(\left(S l_{\alpha}^{-\gamma}\right) l_{\delta}^{+\beta}\right)_{i j} .
$$

For the example of $U_{q}\left(s u_{2}\right)$ one has [Maj95]

$$
l^{+}=\left(\begin{array}{cc}
q^{\frac{H}{2}} & 0 \\
q^{-\frac{1}{2}}\left(q-q^{-1}\right) X_{+} & q^{-\frac{H}{2}}
\end{array}\right), \quad l^{-}=\left(\begin{array}{cc}
q^{-\frac{H}{2}} & q^{\frac{1}{2}}\left(q^{-1}-q\right) X_{-} \\
0 & q^{\frac{H}{2}}
\end{array}\right),
$$

giving the formulae for $x^{\alpha}$ previously used. It seems clear that this calculus will similarly extend to $C_{\mathcal{D}}^{\infty}(\mathbb{G})$ for the general $q$-deformation of a compact simple group with $A=\mathbb{C}[\mathbb{G}]$ coquaistriangular. Details will be considered elsewhere.

\section{Concluding Remarks}

Having a suitable summable $\mathcal{D}$ to define a smooth subspace $C_{\mathcal{D}}^{\infty}$ to which the differential calculus extends, as above, is an important step towards an actual geometric Dirac operator. In the coquasitriangular case with the bicovariant calculus defined by a matrix corepresentation, we have $\Lambda^{1}=\operatorname{End}(V)$ for some comodule $V$ and following [Maj03] we can define 'spinor sections' $S_{\mathcal{D}}^{\infty}=C_{\mathcal{D}}^{\infty} \otimes V$ and a canonical map

$$
D: S_{\mathcal{D}}^{\infty} \rightarrow S_{\mathcal{D}}^{\infty}, \quad D\left(s_{\beta} \otimes e_{\beta}\right)=\partial^{\alpha \beta} s_{\beta} \otimes e_{\alpha}
$$

where $\left\{e_{\alpha}\right\}$ is a basis of $V$ and $s_{\alpha} \in C_{\mathcal{D}}^{\infty}$. At the algebraic level this was

$$
D=(\mathrm{id} \otimes \mathrm{ev})(\mathrm{d} \otimes \mathrm{id}): A \otimes V \rightarrow A \otimes \operatorname{End}(V) \otimes V \rightarrow A \otimes V
$$

in [Maj03], but since the partial derivatives extend to 'smooth functions' we see that so does $D$ to our 'smooth sections'. This was studied at the algebraic level in detail for $A=\mathbb{C}_{q}\left[S U_{2}\right]$ and justified as a natural Dirac-like operator that bypasses the Clifford algebra in the usual construction of the geometric Dirac operator, and fits with that after we add an additional constant curvature term (a multiple of the identity). Using our results for this quantum group we have

$$
D\left(\begin{array}{c}
\alpha t_{m n}^{l} \\
\beta t_{p r}^{l^{\prime}}
\end{array}\right)=\left(\begin{array}{l}
\alpha t_{m s}^{l} \sigma^{11}\left(t_{s n}^{l}\right)+\beta t_{p s}^{l^{\prime}} \sigma^{12}\left(t_{s r}^{l^{\prime}}\right) \\
\alpha t_{m s}^{l} \sigma^{21}\left(t_{s n}^{l}\right)+\beta t_{p s}^{l^{\prime}} \sigma^{22}\left(t_{s r}^{l^{\prime}}\right)
\end{array}\right)
$$

for coefficients $\alpha, \beta$ and for the symbols (7.20) given previously (we sum over $s$ in the appropriate range). The eigenvalues of the geometrically normalised $D / \lambda$ when restricted to both spinor components in the Peter-Weyl subspace spanned by $\left\{t_{m n}^{l}\right\}$ are

$$
\text { (i) } \quad q^{l+1}[l]_{q} ; \quad(\text { ii })(l>0)-q^{-l}[l+1]_{q}
$$

and fully diagonalise this subspace of dimension $2(2 l+1)^{2}$, and hence together fully diagonalise $D$. The type (i) eigenvalues were already noted for the reduced Hopf algebras at odd roots unity in [Maj03, Prop. 5.2] in the equivalent form $q^{2}[2 l ; q] /[2 ; q]=q^{2}\left[l ; q^{2}\right]$, where $[m ; q]=\left(q^{m}-1\right) /(q-1)=1+q+\cdots+q^{m-1}$. We see using our Fourier methods that we also have a second set (ii) both at roots of unity (beyond the 3rd root) and for real or generic $q$. Note that our above geometric $D$ is not directly comparable to our operators $\mathcal{D}$ because our spinor space is two-dimensional so that $D$ does not act on one copy of the coordinate algebra, and nor should it geometrically, but is in the same ball 
park as the $q$-deformation (7.15) of the classical $\mathcal{D}$ with eigenvalues $2 l+1$ discussed in Example 5.6.

Also note that the bicovariant matrix block calculi are typically inner in the sense of a nonclassical direction $\theta$ such that $[\theta, f]=\lambda \mathrm{d} f$, and that is the case for the 4D calculus on $\mathbb{C}_{q}\left[\mathrm{SU}_{2}\right]$ with $\theta=e_{a}+e_{d}$. One can choose a more geometric basis $e_{z}, e_{b}, e_{c}, \theta$ where the first three have a classical limit as usual and $e_{z}=q^{-2} e_{a}-e_{d}$. The partial derivative $\partial^{\theta}$ for the $\theta$-direction in this basis turns out to be the $q$-deformed Laplacian $\Delta_{q}$ as explained in [Maj15]. There is a quantum metric

$$
g=e_{c} \otimes e_{b}+q^{2} e_{b} \otimes e_{c}+\frac{q^{2}}{q+q^{-1}}\left(e_{z} \otimes e_{z}-\theta \otimes \theta\right)
$$

and denoting its coefficients as $g_{i j}$ one has a natural $q$-Laplace operator [Maj15]

$$
\Delta_{q}=\frac{q}{2} g_{i j} \partial^{i} \partial^{j}, \quad \partial^{\theta}=\frac{q \partial^{a}+q^{-1} \partial^{d}}{q+q^{-1}}=\frac{q^{2} \lambda^{2}}{q+q^{-1}} \Delta_{q}
$$

(where we have changed to our more geometric normalisation of $\partial^{i}$ and d). Once again, since we have seen that the partial derivatives extend to $C_{\mathcal{D}}^{\infty}\left(\mathrm{SU}_{2}^{\mathrm{q}}\right)$, this $\Delta_{q}$ also extends and, using our result (7.20), we are in a position to compute it in our Peter-Weyl basis as

$$
\begin{aligned}
\Delta_{q} t_{m n}^{l}=\frac{[2]_{q}}{q^{2} \lambda^{2}} \partial^{\theta} t_{m n}^{l} & =q^{-2} \lambda^{-2}\left(q t_{m s}^{l} \sigma^{11}\left(t_{s n}^{l}\right)+q^{-1} t_{m s}^{l} \sigma^{22}\left(t_{s n}^{l}\right)\right) \\
& =\frac{q\left(q^{2 l}-1\right)+q^{-1}\left(q^{-2 l}-1\right)}{\left(q-q^{-1}\right)^{2}} t_{m n}^{l} \\
& =[l]_{q}[l+1]_{q} t_{m n}^{l}
\end{aligned}
$$

for $m, n=-l, \ldots, l$. One could then take a square root involving $\Delta_{q}$ much as in Example 5.5 for the operator $\mathcal{D}$ to provide the smoothness.

Further $q$-harmonic analysis using our Fourier methods will be considered elsewhere to include smooth functions and harmonic analysis on the $q$-sphere obtained from the $3 \mathrm{D}$ differential calculus on $C_{\mathcal{D}}^{\infty}\left(\mathrm{SU}_{2}^{\mathrm{q}}\right)$, extending the algebraic line for the geometric Dirac operator on the $q$-sphere in [BM15]. Note that our $q$-geometric Dirac operators are not exactly parts of spectral triples in the strict Connes sense, although the one on the $q$-sphere comes close at the algebraic level. The bounded commutator issue was already noted at the end of Sect. 5 for the $q$-deformed $\mathcal{D}$ in (7.15). In [KS12] it is shown for $\mathrm{SU}_{2}^{\mathrm{q}}$ that an operator with similar eigenvalues to the above $D$ has bounded 'commutator' provided the latter is twisted by the left modular automorphism. Investigation of the precise relationship between algebro-geometric triples, such as in [Maj03,BM15], and twisted spectral triples [Con08], should be an interesting topic for further work.

Open Access This article is distributed under the terms of the Creative Commons Attribution 4.0 International License (http://creativecommons.org/licenses/by/4.0/), which permits unrestricted use, distribution, and reproduction in any medium, provided you give appropriate credit to the original author(s) and the source, provide a link to the Creative Commons license, and indicate if changes were made. 


\section{References}

[ANR15a] Akylzhanov, R., Nursultanov, E., Ruzhansky, M.: Hardy-Littlewood, Hausdorff-Young-Paley inequalities, and $L^{p}-L^{q}$ multipliers on compact homogeneous manifolds. arXiv:1504.07043, (2015)

[ANR15b] Akylzhanov, K.R., Nursultanov, D.E., Ruzhanskiĭ, V.M.: Hardy-Littlewood-Paley-type inequalities on compact Lie groups. Mat. Zametki 100(2), 287-290 (2016).

[ANR16] Akylzhanov, R., Nursultanov, E., Ruzhansky, M.: Hardy-Littlewood-Paley inequalities and Fourier multipliers on $S U(2)$. Studia Math. 234(1), 1-29 (2016)

[AR16] Akylzhanov, R., Ruzhansky, M.: Fourier multipliers and group von Neumann algebras. C. R. Math. Acad. Sci. Paris 354(8), 766-770 (2016)

[BC12] Bekjan, N.T., Chen, Z.: Interpolation and $\phi$-moment inequalities of noncommutative martingales. Probab. Theory Relat. Fields 152(1), 179-206 (2012)

[BL76] Bergh, J., Löfström, J.: Interpolation spaces. An introduction. Springer-Verlag, Berlin-New York. Grundlehren der Mathematischen Wissenschaften, No. 223 (1976)

[BM15] Beggs, E., Majid, S.: Spectral triples from bimodule connections and Chern connections. J. Noncommut. Geom. 11(2), 669-701 (2015)

[CP08] Chakraborty, S.P., Pal, A.: Characterization of $\mathrm{SU}_{q}(l+1)$-equivariant spectral triples for the odd dimensional quantum spheres. J. Reine Angew. Math. 623, 25-42 (2008)

[CFK14] Cipriani, F., Franz, U., Kula, A.: Symmetries of Lévy processes on compact quantum groups, their Markov semigroups and potential theory. J. Funct. Anal. 266(5), 2789-2844 (2014)

[CL01] Connes, A., Landi, G.: Noncommutative manifolds, the instanton algebra and isospectral deformations. Comm. Math. Phys. 221(1), 141-159 (2001)

[Con95] Connes, A.: Noncommutative geometry and reality. J. Math. Phys. 36(11), 6194-6231 (1995)

[Con96] Connes, A.: Gravity coupled with matter and the foundation of non-commutative geometry. Commun. Math. Phys. 182(1), 155-176 (1996)

[Con08] Connes, A., Moscovici, H.: Type III and spectral triples. In: Traces in Number Theory, Geometry and Quantum Fields, Aspects Math., E38, pp. 57-71. Friedr. Vieweg, Wiesbaden (2008)

[Con13] Connes, A.: On the spectral characterization of manifolds. J. Noncommut. Geom. 7, 1-82 (2013)

[Coo10] Cooney, T.: A Hausdorff-Young inequality for locally compact quantum groups. Int. J. Math. 21(12), 1619-1632 (2010)

[DLS ${ }^{+}$05] Dabrowski, L., Landi, G., Sitarz, A., Suijlekom, W.van , Värilly, C.J.: The Dirac operator on $S U_{2}^{q}$. Commun. Math. Phys. 259(3), 729-759 (2005)

[DK94] Dijkhuizen, S.M., Koornwinder, H.: $\mathbb{C}_{q}[\mathfrak{g}]$ algebras: a direct algebraic approach to compact quantum groups. Lett. Math. Phys. 32(4), 315-330 (1994)

[FGV00] Figueroa, H., Gracia-Bondia, J., Varilly, J.: Elements of Noncommutative Geometry. Birkhäuser, New York (2000)

[Fio98] Fiore, G.: Deforming maps for Lie group covariant creation and annihilation operators. J. Math. Phys. 39(6), 3437-3452 (1998)

[Fol99] Folland, G.B.: Real analysis. Pure and Applied Mathematics (New York). John Wiley \& Sons, Inc., New York, second edition. Modern techniques and their applications, A Wiley-Interscience Publication (1999)

[Gro55] Grothendieck, A.: Produits tensoriels topologiques et espaces nucléaires. Mem. Amer. Math. Soc. 16, 140 (1955)

[Haa79] Haagerup, U.: $L^{p}$-spaces associated with an arbitrary von Neumann algebra. In Algèbres d'opérateurs et leurs applications en physique mathématique (Proc. Colloq., Marseille, 1977), volume 274 of Colloq. Int. CNRS, pp. 175-184. CNRS, Paris (1979)

[HL36] Hardy, G., Littlewood, J.: Some theorems concerning Fourier series and Fourier power series. Duke Math. J. 2, 354-382 (1936)

[HR74] Hewitt, E., Ross, A.K.: Rearrangements of $L^{r}$ Fourier series on compact Abelian groups. Proc. Lond. Math. Soc. (3) 29, 317-330 (1974)

[JNR09] Junge, M., Neufang, M., Ruan, Z.-J.: A representation theorem for locally compact quantum groups. Int. J. Math. 20(3), 377-400 (2009)

[KS12] Kaad, J., Senior, R.: A twisted spectral triple for quantum SU(2). J. Geom. Phys. 62(4), 731739 (2012)

[Kos84] Kosaki, H.: Applications of the complex interpolation method to a von Neumann algebra: noncommutative $L^{p}$-spaces. J. Funct. Anal. 56(1), 29-78 (1984)

[LNJP16] Lévy, C., Neira Jiménez, C., Paycha, S.: The canonical trace and the noncommutative residue on the noncommutative torus. Trans. Amer. Math. Soc. 368(2), 1051-1095 (2016)

[Lic90] Lichnerowicz, A.: Spineurs harmoniques et spineurs-twisteurs en géométrie kählerienne et conformément kählerienne. C. R. Math. Acad. Sci. Paris. 311(13), 883-887 (1990)

[LWW17] Liu, Z., Wang, S., Wu, J.: Young's inequality for locally compact quantum groups. J. Op. Theory $77(1), 109-131(2017)$ 
[Maj95] Majid, S.: Foundations of Quantum Group Theory. Cambridge University Press, Cambridge (1995)

[Maj03] Majid, S.: Noncommutative Ricci curvature and Dirac operator on $\mathrm{C}_{q}\left[\mathrm{SL}_{2}\right]$ at roots of unity. Lett. Math. Phys. 63(1), 39-54 (2003)

[Maj15] Majid, S.: Hodge star as braided Fourier transform. Algebr. Represent. Theory 20, 695-733 (2017)

[Maj16] Majid, S.: Noncommutative differential geometry. In: Bullet T.F.S., Smith, F. (eds) LTCC Lecture Notes Series: Analysis and Mathematical Physics, pp. 139-176. World Scientific, Singapore (2017)

[MMN $\left.{ }^{+} 91\right]$ Masuda, T., Mimachi, K., Nakagami, Y., Noumi, M., Ueno, K.: Representations of the quantum group $S U_{q}(2)$ and the little $q$-Jacobi polynomials. J. Funct. Anal. 99(2), 357-386 (1991)

[MVD98] Maes, A., Van Daele, A.: Notes on compact quantum groups. Nieuw Arch. Wisk. (4). 16(1-2), 73112 (1998)

[NT10] Neshveyev, S., Tuset, L.: The Dirac operator on compact quantum groups. J. Reine Angew. Math. 2010(641), 1-20 (2010)

[RT10] Ruzhansky, M., Turunen, V.: Pseudo-differential operators and symmetries, volume 2 of PseudoDifferential Operators. Theory and Applications. Birkhäuser Verlag, Basel. Background analysis and advanced topics. (2010)

[RT13] Ruzhansky, M., Turunen, V.: Global quantization of pseudo-differential operators on compact Lie groups, SU(2), 3-sphere, and homogeneous spaces. Int. Math. Res. Not. IMRN. 11, 24392496 (2013)

[RT16] Ruzhansky, M., Tokmagambetov, N.: Nonharmonic analysis of boundary value problems. Int. Math. Res. Not. IMRN. 12, 3548-3615 (2016)

[TK86] Thierry, F., Kosaki, H.: Generalized $s$-numbers of $\tau$-measurable operators. Pac. J. Math. 123(2), 269300 (1986)

[Tre67] Trèves, F.: Topological Vector Spaces, Distributions and Kernels. Academic Press, New York, London (1967)

[Tri78] Triebel, H.: Interpolation Theory, Function Spaces, Differential Operators, volume 18 of NorthHolland Mathematical Library. North-Holland Publishing Co., Amsterdam, New York (1978)

[Wor87] Woronowicz, L.S.: Compact matrix pseudogroups. Comm. Math. Phys. 111(4), 613-665 (1987)

[Wor89] Woronowicz, L.S.: Differential calculus on compact matrix pseudogroups (quantum groups). Comm. Math. Phys. 122(1), 125-170 (1989)

[Wor98] Woronowicz, L.S.: Compact quantum groups. In: Symétries Quantiques (Les Houches, 1995), pp. 845-884. North-Holland, Amsterdam (1998)

[Xu07] Xu, Q.: Operator spaces and noncommutative lp. The part on non-commutative Lp-spaces. Lectures in the Summer School on Banach spaces and Operator spaces, Nankai University-China (2007)

[You08] Youn, S.-G.: Hardy-Littlewood inequalities on compact quantum groups of Kac type. Anal. PDE. 11(1), 237-261 (2018)

Communicated by C. Schweigert 